\title{
Interval-valued Atanassov intuitionistic OWA aggregations using admissible linear orders and their application to decision making
}

\author{
Laura De Miguel, Humberto Bustince, Senior Member, IEEE, Barbara Pȩkala, Urszula Bentkowska, \\ Ivanosca Da Silva, Benjamin Bedregal Member, IEEE, Radko Mesiar and Gustavo Ochoa
}

\begin{abstract}
Based on the definition of admissible order for interval-valued Atanassov intuitionistic fuzzy sets, we study OWA operators in these sets distinguishing between the weights associated to the membership and those associated to the nonmembership degree which may differ from the latter. We also study Choquet integrals for aggregating information which is represented using interval-valued Atanassov intuitionistic fuzzy sets. We conclude with two algorithms to choose the best alternative in a decision making problem when we use this kind of sets to represent information.
\end{abstract}

Index Terms-Interval-valued Atanassov intuitionistic fuzzy set; interval-valued Atanassov intuitionistic OWA operator; Unbalanced interval-valued Atanassov intuitionistic OWA operator; interval-valued Atanassov intuitionistic Choquet Integral.

\section{INTRODUCTION}

OWA operators [1] and Choquet integrals [2] are often used for fusing information. The goal of this paper is to extend these operators for using Interval-Valued Atanassov Intuitionistic Fuzzy Sets (IVAIFSs)[3] to represent the information. To achieve this goal, we focus on the definition and construction of a particular class of linear orders on IVAIFSs called admissible and defined in [4].

We know that, in order to aggregate information represented by means of IVAIFSs, we should aggregate the intervals which represent the membership, on one hand, and the intervals which represent the non-membership, on the other hand. This fact has suggested us to propose the use of Unbalanced OWA operators, in the sense that we use admissible orders and different weight vectors for aggregating the membership and the non-membership values.

Laura De Miguel and Humberto Bustince are with the Departamento de Automática y Computación and with Institute of Smart Cities, Universidad Publica de Navarra, Campus Arrosadia s/n, P.O. Box 31006, Pamplona, Spain.

Ivanoska Da Silva is with the Instituto Metropole Digital, Universidade Federal do Rio Grande do Norte, Campus Universitário s/n, 59072-970 Natal, Brazil.

Benjamin Bedregal is with the Departamento de Informática e Matemática Aplicada, Universidade Federal do Rio Grande do Norte, Campus Universitário s/n, 59072-970 Natal, Brazil.

Barbara Pȩkala and Urszula Bentkowska are with Faculty of Mathematics and Natural Sciences, University of Rzeszów, Al. Rejtana 16a, 35-959 Rzeszów, Poland.

Radko Mesiar is with Faculty of Civil Engineering, Slovak University of Technology in Bratislava, Radlinskeho 9-11, Bratislava, Slovakia and also with the Institute for Research and Applications of Fuzzy Modeling, University of Ostrava, 70103 Ostrava, Czech Republic.

Gustavo Ochoa is with the Departamento de Matematicas, Universidad Publica de Navarra, Campus Arrosadia s/n, P.O. Box 31006, Pamplona, Spain.
Besides, it is known that for some decision making problems, experts may have problems to provide exact numerical values to represent their preferences and non-preferences between the different alternatives. In these cases, some authors advise for the use of intervals ([5], [6], [7], [8]) to represent such preferences and non-preferences. In this situation, a suitable option is to represent the information by means of IVAIFSs. To do so, it is necessary an appropriate theoretical development of aggregation functions such as OWAs and Choquet integrals [9]. We discuss the usefulness of our theoretical developments in the last part of this work, where we present two algorithms to select the best alternative in a multi-expert decision making problem where the preferences are given as IVAIFSs.

This work is organized as follows. In Preliminaries we discuss several concepts which are going to be used along the paper. In Section III, we recall the definition and two methods of construction of interval-valued Atanassov intuitionistic fuzzy admissible orders using aggregation functions. Next, we develop OWA operators for interval-valued Atanassov intuitionistic vectors in Section IV while in Section V we propose Unbalanced OWA operators for IVAIFSs. In Section VI, we introduce the discrete Choquet integral for these sets and we present two algorithms which make use of admissible orders, OWA operators and Choquet integrals when we are dealing with IVAIFSs in a multi-expert decision making problem in Section VII. We finish with some concluding remarks and references.

\section{Preliminaries}

In this section we introduce some preliminary notions in order to fix notation. We denote $L([0,1])$ to the set of all closed subintervals of the unit interval, that is,

$$
L([0,1])=\left\{\mathbf{x}=\left[\underline{x_{1}}, \overline{x_{1}}\right] \mid 0 \leq \underline{x_{1}} \leq \overline{x_{1}} \leq 1\right\} .
$$

Since Zadeh [10] introduced the concept of fuzzy sets different generalizations have been defined, see [11]. In particular, Interval-Valued Atanassov Intuitionistic Fuzzy Sets (IVAIFSs) generalize the concept of intuitionistic fuzzy sets given in 1986 [12] by Atanassov. In this work we introduce the notions of OWA operator and discrete Choquet integral for IVAIFSs. Some other studies on these sets are [13], [14], [15]. 
Definition 2.1: ([3]) Let $U \neq \emptyset$ be a universe. An IntervalValued Atanassov Intuitionistic Fuzzy Set (IVAIFS) $G$ over $U$ is the set

$$
G=\left\{\left(u, \mathbf{x}_{u}, \mathbf{y}_{u}\right) \mid u \in U\right\}
$$

where $\mathbf{x}_{u}=[x(u), \overline{x(u)}], \mathbf{y}_{u}=[y(u), \overline{y(u)}] \in L([0,1])$ state, respectively, the membership degree and the nonmembership degree of $u$ to $G$ and they satisfy that for all $u \in U, \overline{x(u)}+$ $\overline{y(u)} \leq 1$

Pairs $\left(\mathbf{x}_{u}, \mathbf{y}_{u}\right)$ are called Interval-Valued Atanassov Intuitionistic Fuzzy pairs (IVAIF-pairs), while the set of all possible IVAIF-pairs is denoted

$$
\mathcal{L}_{I V}([0,1])=\{z=(\mathbf{x}, \mathbf{y}) \mid \mathbf{x}, \mathbf{y} \in L([0,1]) \text { and } \bar{x}+\bar{y} \leq 1\} .
$$

Given an IVAIFS $G, z_{G}(u)=\left(\mathbf{x}_{G, u}, \mathbf{y}_{G, u}\right)$ denotes the IVAIF-pair associated with the referential element $u \in U$. However, for the sake of simplicity, $z_{G}(u)$ is abbreviated to $z=(\mathbf{x}, \mathbf{y})$ when possible.

Definition 2.2: Let $G_{1}, G_{2}$ be two IVAIFSs. Atanassov proposed an order $\leq$ such that $G_{1} \leq G_{2}$ if and only if, for all $u \in U$,

$$
\mathbf{x}_{G_{1}, u} \preceq_{2} \mathbf{x}_{G_{2}, u} \text { and } \mathbf{y}_{G_{1}, u} \succeq_{2} \mathbf{y}_{G_{2}, u},
$$

where $\preceq_{2}$ is the partial order on $L([0,1])$, given by

$$
\left[\underline{p_{1}}, \overline{p_{1}}\right] \preceq_{2}\left[\underline{p_{2}}, \overline{p_{2}}\right] \text { if and only if } \underline{p_{1}} \leq \underline{p_{2}} \text { and } \overline{p_{1}} \leq \overline{p_{2}} \text {. }
$$

Aggregation functions [16], [17] are a frequently used tool in fuzzy logic with their extensions and their applications.

Definition 2.3: Let $(P, \preceq)$ be a Partially Ordered Set (i.e., a poset) with bottom $0_{P}$ and top $1_{P}$. An n-aggregation function with respect to the order $\preceq$ is a mapping $M: P^{n} \rightarrow P$ such that

$$
\begin{aligned}
& \text { - } M\left(0_{P}, \ldots, 0_{P}\right)=0_{P} \text { and } M\left(1_{P}, \ldots, 1_{P}\right)=1_{P}, \\
& \text { - if }\left(x_{1}, \ldots, x_{n}\right) \preceq\left(y_{1}, \ldots, y_{n}\right), \\
& \text { then } M\left(x_{1}, \ldots, x_{n}\right) \preceq M\left(y_{1}, \ldots, y_{n}\right),
\end{aligned}
$$

where $\left(x_{1}, \ldots, x_{n}\right) \preceq\left(y_{1}, \ldots, y_{n}\right)$ if and only if $x_{i} \preceq y_{i}$ for all $i \in\{1, \ldots, n\}$.

Note that Definition 2.3 extends the definition of aggregation operator on the unit interval $[0,1]$ (see [18]). In particular, in this work we study some specific aggregation functions that are OWA operators and Choquet integrals.

Definition 2.4: [1] Let $w=\left(w_{1}, \ldots, w_{n}\right) \in[0,1]^{n}$ with $w_{1}+\ldots+w_{n}=1$. The Ordered Weighted Averaging (OWA) operator associated with $w$ is a mapping $O W A_{w}:[0,1]^{n} \longrightarrow$ $[0,1]$ given by

$$
O W A_{w}\left(x_{1}, \ldots, x_{n}\right)=\sum_{i=1}^{n} w_{i} x_{(i)}
$$

where $x_{(i)}, i \in\{1, \ldots, n\}$, denotes the $i$-th greatest component of $\left(x_{1}, \ldots, x_{n}\right)$.

Definition 2.5: Let $U \neq \emptyset$ be a finite universe. A fuzzy measure $m$ is a mapping $m: 2^{U} \longrightarrow[0,1]$ such that $m(\emptyset)=$ $0, m(U)=1$ and $m\left(F_{1}\right) \leq m\left(F_{2}\right)$ whenever $F_{1} \subset F_{2}$ for $F_{1}, F_{2} \in 2^{U}$.

Definition 2.6: Let $m$ be a fuzzy measure on a non-empty finite universe $U=\left\{u_{1}, \ldots, u_{n}\right\} \neq \emptyset$. The discrete Choquet integral of a fuzzy set $\mu: U \longrightarrow[0,1]$ with respect to $m$ is defined as

$$
\begin{aligned}
C_{m}(\mu)=\sum_{i=1}^{n} \mu\left(u_{\sigma(i)}\right) & m\left(\left\{u_{\sigma(i)}, \ldots, u_{\sigma(n)}\right\}\right) \\
& -\mu\left(u_{\sigma(i)}\right) m\left(\left\{u_{\sigma(i+1)}, \ldots, u_{\sigma(n)}\right\}\right),
\end{aligned}
$$

where $\sigma$ is a permutation such that $\mu\left(u_{\sigma(1)}\right) \leq \mu\left(u_{\sigma(2)}\right) \leq$ $\ldots \leq \mu\left(u_{\sigma(n)}\right)$ and, $m\left(\left\{u_{\sigma(n+1)}, u_{\sigma(n)}\right\}\right)=0$ by convention.

As defined in [19], a fuzzy measure is called symmetric if $m(F)$ depends only on the cardinality of the set $F \subseteq U$, i.e., for any $F_{1}, F_{2} \subseteq U$ if $\left|F_{1}\right|=\left|F_{2}\right|$ then $m\left(F_{1}\right)=m\left(F_{2}\right)$. OWA operators are a special class of Choquet integrals where the fuzzy measure associated is symmetric.

A relevant characteristic of OWA operators and Choquet integrals is the fact that they require a linear order on the set of inputs. Hence, although the definition of aggregation function is grounded on partial orders, linear orders play a very relevant role in the generation of some of these operators. In the development of the paper, some results of [9], [20] are applied.

An order $\leq$ on $L([0,1])$ is called admissible if it is linear and satisfies that, for all $\mathbf{x}, \mathbf{y} \in L([0,1])$, such that $\mathbf{x} \preceq_{2} \mathbf{y}$ then $\mathbf{x} \leq \mathbf{y}$ [20].

Proposition 2.1: [20] Let $B_{1}, B_{2}:[0,1]^{2} \mapsto[0,1]$ be two continuous aggregation functions, such that, for all $\mathbf{x}=$ $[\underline{x}, \bar{x}], \mathbf{y}=[y, \bar{y}] \in L([0,1])$, the equalities $B_{1}(\underline{x}, \bar{x})=$ $B_{1}(y, \bar{y})$ and $\bar{B}_{2}(\underline{x}, \bar{x})=B_{2}(y, \bar{y})$ hold if and only if $\mathbf{x}=\mathbf{y}$.

If the order $\leq_{B_{1,2}}$ on $L([0, \overline{1}])$ is defined by $\mathbf{x} \leq_{B_{1,2}} \mathbf{y}$ if and only if

$$
\begin{aligned}
B_{1}(\underline{x}, \bar{x})<B_{1}(\underline{y}, \bar{y}) \quad \text { or } \\
\quad\left(B_{1}(\underline{x}, \bar{x})=B_{1}(\underline{y}, \bar{y}) \text { and } B_{2}(\underline{x}, \bar{x}) \leq B_{2}(\underline{y}, \bar{y})\right)
\end{aligned}
$$

then $\leq_{B_{1,2}}$ is an admissible order on $L([0,1])$.

In [9], this class of linear orders on $L([0,1])$ is used to extend the definition of OWA operators for interval-valued fuzzy sets.

Definition 2.7: Let $\leq$ be an admissible order on $L([0,1])$, and let $w=\left(w_{1}, \ldots, w_{n}\right) \in[0,1]^{n}$, with $w_{1}+\ldots+w_{n}=1$. The Interval-Valued OWA operator associated with $\leq$ and $w$ is a mapping $I V O W A_{[\leq, w]}:\left(L([0,1])^{n} \longrightarrow L([0,1])\right.$ given by

$$
\operatorname{IVOW} A_{[\leq, w]}\left(\left[a_{1}, b_{1}\right], \ldots,\left[a_{n}, b_{n}\right]\right)=\sum_{i=1}^{n} w_{i} \cdot\left[a_{(i)}, b_{(i)}\right]
$$

where $\left[a_{(i)}, b_{(i)}\right], i=1, \ldots, n$ denotes the $i$-th greatest of the inputs with respect to the order $\leq$, and the operations are $w \cdot[a, b]=[w a, w b]$ and $\left[x_{1}, y_{1}\right]+\left[x_{2}, y_{2}\right]=\left[x_{1}+x_{2}, y_{1}+y_{2}\right]$.

\section{IVAIF-ADMISSIBLE ORDERS}

Since the aim of the paper is to define OWA operators and Choquet Integrals for IVAIFS, the generation of linear orders plays a crucial role. The linear orders we based on the rest of the work were presented in [4] where a study on linear orders similar to the previous ones on $L([0,1])$ [9], but focused on IVAIFS is done. 
An IVAIF-admissible order $\leq$ on $\mathcal{L}_{I V}([0,1])$ is a linear order which refines Atanassov's partial order in Eq. (1). We recall a method to construct IVAIF-admissible orders [4] .

Proposition 3.1: Let $B=\left\langle B_{1}, B_{2}, B_{3}, B_{4}\right\rangle$ be a set of four aggregation functions, $B_{i}:[0,1]^{2} \longrightarrow[0,1]$ such that they generate admissible orders $\leq_{B_{1,2}}, \leq_{B_{3,4}}$ on $L([0,1])$ as in the Proposition 2.1. An IVAIF-admissible order $\leq_{B}$ on $(L([0,1]))^{2}$ is such that $\left(\mathbf{x}_{1}, \mathbf{y}_{1}\right) \leq_{B}\left(\mathbf{x}_{2}, \mathbf{y}_{2}\right)$ if an only if

$$
\mathbf{x}_{1}<_{B_{1,2}} \mathbf{x}_{2} \quad \text { or } \quad\left(\mathbf{x}_{1}={ }_{B_{1,2}} \mathbf{x}_{2} \text { and } \mathbf{y}_{1} \geq_{B_{3,4}} \mathbf{y}_{2}\right) .
$$

In particular, if $B_{1}=B_{3}$ and $B_{2}=B_{4}$, the orders $\leq_{B_{1,2}}$ and $\leq_{B_{3,4}}$ are equal: the same order is used to compare both intervals although in the second one the order is reversed.

Proposition 3.2: Let $A=\left\langle A_{1}, A_{2}, A_{3}, A_{4}\right\rangle$ be a set of four aggregation functions, $A_{i}:[0,1]^{4} \rightarrow[0,1]$ such that, for all $\left(p_{1}, q_{1}, r_{1}, s_{1}\right),\left(p_{2}, q_{2}, r_{2}, s_{2}\right) \in[0,1]^{4}$ the equalities $A_{i}\left(p_{1}, q_{1}, r_{1}, s_{1}\right)=A_{i}\left(p_{2}, q_{2}, r_{2}, s_{2}\right)$ for all $i \in\{1, \ldots, 4\}$ only hold simultaneously if $\left(p_{1}, q_{1}, r_{1}, s_{1}\right)=\left(p_{2}, q_{2}, r_{2}, s_{2}\right)$.

Then, the relation $\left(\mathbf{x}_{1}, \mathbf{y}_{1}\right) \leq_{A}\left(\mathbf{x}_{2}, \mathbf{y}_{2}\right)$ if and only if one of the (mutually exclusive) conditions is satisfied

i) $A_{1}\left(\underline{x}_{1}, \bar{x}_{1}, 1-\underline{y}_{1}, 1-\bar{y}_{1}\right)<A_{1}\left(\underline{x}_{2}, \bar{x}_{2}, 1-\underline{y}_{2}, 1-\bar{y}_{2}\right)$.

ii) $A_{1}\left(\underline{x}_{1}, \bar{x}_{1}, 1-\underline{y}_{1}, 1-\bar{y}_{1}\right)=A_{1}\left(\underline{x}_{2}, \bar{x}_{2}, 1-\underline{y}_{2}, 1-\bar{y}_{2}\right)$ and

$A_{2}\left(\underline{x}_{1}, \bar{x}_{1}, 1-\underline{y}_{1}, 1-\bar{y}_{1}\right)<A_{2}\left(\underline{x}_{2}, \bar{x}_{2}, 1-\underline{y}_{2}, 1-\bar{y}_{2}\right)$.

iii) $A_{1}\left(\underline{x}_{1}, \bar{x}_{1}, 1-\underline{y}_{1}, 1-\bar{y}_{1}\right)=A_{1}\left(\underline{x}_{2}, \bar{x}_{2}, 1-\underline{y}_{2}, 1-\bar{y}_{2}\right)$, $A_{2}\left(\underline{x}_{1}, \bar{x}_{1}, 1-\underline{y}_{1}, 1-\bar{y}_{1}\right)=A_{2}\left(\underline{x}_{2}, \bar{x}_{2}, 1-\underline{y}_{2}, 1-\bar{y}_{2}\right)$ and

$A_{3}\left(\underline{x}_{1}, \bar{x}_{1}, 1-\underline{y}_{1}, 1-\bar{y}_{1}\right)<A_{3}\left(\underline{x}_{2}, \bar{x}_{2}, 1-\underline{y}_{2}, 1-\bar{y}_{2}\right)$.

iv) $A_{1}\left(\underline{x}_{1}, \bar{x}_{1}, 1-\underline{y}_{1}, 1-\bar{y}_{1}\right)=A_{1}\left(\underline{x}_{2}, \bar{x}_{2}, 1-\underline{y}_{2}, 1-\bar{y}_{2}\right)$, $A_{2}\left(\underline{x}_{1}, \bar{x}_{1}, 1-\underline{y}_{1}, 1-\bar{y}_{1}\right)=A_{2}\left(\underline{x}_{2}, \bar{x}_{2}, 1-\underline{y}_{2}, 1-\bar{y}_{2}\right)$, $A_{3}\left(\underline{x}_{1}, \bar{x}_{1}, 1-\underline{y}_{1}, 1-\bar{y}_{1}\right)=A_{3}\left(\underline{x}_{2}, \bar{x}_{2}, 1-\underline{y}_{2}, 1-\bar{y}_{2}\right)$ and

$A_{4}\left(\underline{x}_{1}, \bar{x}_{1}, 1-\underline{y}_{1}, 1-\bar{y}_{1}\right) \leq A_{4}\left(\underline{x}_{2}, \bar{x}_{2}, 1-\underline{y}_{2}, 1-\bar{y}_{2}\right)$

is an IVAIF-admissible order on $\mathcal{L}_{I V}([0,1])$.

Example 3.3: Particular examples of $\leq_{A}$ orders are the following.

1) Let $Q_{i}$ refer to an aggregation function given by

$$
Q_{i}\left(\underline{x}_{1}, \bar{x}_{1}, \underline{y}_{1}, \bar{y}_{1}\right)=a_{i} \underline{x}+b_{i} \bar{x}+c_{i} \underline{y}+d_{i} \bar{y},
$$

such that $a_{i}, b_{i}, c_{i}, d_{i} \geq 0, a_{i}, b_{i}, c_{i}, d_{i} \geq 0, a_{i}+b_{i}+$ $c_{i}+d_{i}=1$. Let $A=\left\langle Q_{1}, Q_{2}, Q_{3}, Q_{4}\right\rangle$ be the set of four of such aggregation functions satisfying

$$
|D|=\left|\begin{array}{llll}
a_{1} & b_{1} & c_{1} & d_{1} \\
a_{2} & b_{2} & c_{2} & d_{2} \\
a_{3} & b_{3} & c_{3} & d_{3} \\
a_{4} & b_{4} & c_{4} & d_{4}
\end{array}\right| \neq 0
$$

Since the aggregation functions satisfy the conditions of Proposition 3.2, they generate an IVAIF-admissible order. We denote this particular class of orders by $\leq_{Q}$.

2) Let $i \in\{1,2\}, j \in\{3,4\}$ and $k_{i}, k_{j} \in[0,1]$. Then the functions:

- $K_{i}(\underline{x}, \bar{x}, \underline{y}, \bar{y})=\underline{x}+k_{i}(\bar{x}-\underline{x}), i \in\{1,2\}$ and

- $K_{j}(\underline{x}, \bar{x}, \bar{y}, \bar{y})=\underline{y}+k_{j}(\bar{y}-\underline{y}), j \in\{3,4\}$

are aggregation functions since $\underline{x} \leq \bar{x}$ and $\underline{y} \leq \bar{y}$.
The orders generated by sets of the type $A=$ $\left\langle K_{1}, K_{2}, K_{3}, K_{4}\right\rangle$ are particular instances of $\leq_{Q}$ orders where

$$
D=\left(\begin{array}{cccc}
1-k_{1} & k_{1} & 0 & 0 \\
1-k_{2} & k_{2} & 0 & 0 \\
0 & 0 & 1-k_{3} & k_{3} \\
0 & 0 & 1-k_{4} & k_{4}
\end{array}\right)
$$

whose determinant is $|D|=\left(k_{2}-k_{1}\right)\left(k_{4}-k_{3}\right)$. If $k_{1} \neq$ $k_{2}$ and $k_{3} \neq k_{4}$ then the aggregation functions satisfy the conditions of the Proposition 3.2 and they generate an IVIAIF-admissible order.

We denote this particular class of orders by $\leq_{K}$.

3) Let $\Pi_{i}$ refer to the $i$-th projection of a 4-place vector. A general lexicographic order, denoted by $\leq_{\Pi}$ is constructed from the four projections. For instance:

- the lexicographic 1 order is generated by the set $A=\left\langle\Pi_{1}, \Pi_{2}, \Pi_{3}, \Pi_{4}\right\rangle$ that we denote with $\leq_{\Pi_{1}}$;

- the composed lexicographic 2 order is generated by the set $A=\left\langle\Pi_{2}, \Pi_{1}, \Pi_{4}, \Pi_{3}\right\rangle$ that we denote with $\leq_{\Pi_{2}}$.

Notice that also the projections are particular instances of $K_{i}$ or $K_{j}$ aggregation functions for $k_{i}, k_{j} \in\{0,1\}$. Thereby, the determinant of $|D|$ is 1 or -1 since each row and each column of the matrix $D$ is generated by all zeros except one element whose value is 1 . For instance, the matrix $D$ associated with the composed lexicographic 2 order is

$$
D=\left(\begin{array}{llll}
0 & 1 & 0 & 0 \\
1 & 0 & 0 & 0 \\
0 & 0 & 0 & 1 \\
0 & 0 & 1 & 0
\end{array}\right)
$$

\section{INTERVAL-VALUED ATANASSOV'S INTUITIONISTIC OWA OPERATORS}

The original definition of OWA operator was given by Yager [1]. In this section we generalize this definition on interval-valued Atanassov intuitionistic fuzzy sets using IVAIF-admissible orders and we study under which conditions they satisfy monotonicity.

Definition 4.1: Let $\leq$ be an IVAIF-admissible order on $\mathcal{L}_{I V}([0,1])$ and let $w=\left(w_{1}, \ldots, w_{n}\right) \in[0,1]^{n}$, with $w_{1}+$ $\ldots+w_{n}=1$. The Interval-Valued Atanassov's Intuitionistic OWA (IVAIOWA) operator associated with $w$ and $\leq$ is a mapping $\left(\mathcal{L}_{I V}([0,1])\right)^{n} \mapsto \mathcal{L}_{I V}([0,1])$ defined by

$$
\begin{aligned}
I V A I O W A_{[w, \leq]}\left(z_{1}, \ldots, z_{n}\right)=\sum_{i=1}^{n} w_{i} \cdot z_{(i)} \\
=\left(\sum_{i=1}^{n} w_{i} \cdot \mathbf{x}_{(i)}, \sum_{i=1}^{n} w_{i} \cdot \mathbf{y}_{(i)}\right) \\
=\left(\sum_{i=1}^{n} w_{i} \cdot\left[\underline{x}_{(i)}, \bar{x}_{(i)}\right], \sum_{i=1}^{n} w_{i} \cdot\left[\underline{y}_{(i)}, \bar{y}_{(i)}\right]\right)
\end{aligned}
$$

where $z_{(i)}$ denotes the $i$-th greatest IVAIF-pair of the inputs $\left(z_{1}, \ldots, z_{n}\right)$ with respect to the order $\leq$ on $\mathcal{L}_{I V}([0,1])$ and 
the interval product and sum are the same as used in Definition 2.7 .

Notice that $w_{1} \underline{x}_{(1)}+\ldots+w_{n} \underline{x}_{(n)} \leq w_{1}+\ldots+w_{n}=1$ (indeed, the same holds for $\bar{x}, y$, and $\bar{y}$ ). The monotonicity of real-valued weighted arithmetic means ensures that each of the components of the result yield by an IVAIOWA is an interval. In addition, the result is always an IVAIF-pair, since

$$
\sum_{i=1}^{n} w_{i} \bar{x}_{(i)}+\sum_{i=1}^{n} w_{i} \bar{y}_{(i)}=\sum_{i=1}^{n} w_{i}\left(\bar{x}_{(i)}+\bar{y}_{(i)}\right) \leq \sum_{i=1}^{n} w_{i}=1
$$

Example 4.1:

Let $w=(0.15,0.35,0.5)$ and let $\leq_{Q}$ be the order generated by the set $A=\left\langle Q_{1}, Q_{2}, Q_{3}, Q_{4}\right\rangle$ as in the Example 3.3 with

- $Q_{1}\left(\underline{x}_{1}, \bar{x}_{1}, \underline{y}_{1}, \bar{y}_{1}\right)=\frac{3}{8} \underline{x}_{1}+\frac{3}{8} \bar{x}_{1}+\frac{1}{8} \underline{y}_{1}+\frac{1}{8} \bar{y}_{1}$,

- $Q_{2}\left(\underline{x}_{1}, \bar{x}_{1}, \underline{y}_{1}, \bar{y}_{1}\right)=\frac{10}{20} \underline{x}_{1}+\frac{5}{20} \bar{x}_{1}+\frac{3}{20} \underline{y}_{1}+\frac{2}{20} \bar{y}_{1}$,

- $Q_{3}\left(\underline{x}_{1}, \bar{x}_{1}, \underline{y}_{1}, \bar{y}_{1}\right)=\frac{1}{20} \underline{x}_{1}+\frac{1}{20} \bar{x}_{1}+\frac{8}{20} \underline{y}_{1}+\frac{1}{20} \bar{y}_{1}$,

- $Q_{4}\left(\underline{x}_{1}, \bar{x}_{1}, \underline{y}_{1}, \bar{y}_{1}\right)=\frac{1}{4} \underline{x}_{1}+\frac{1}{4} \bar{x}_{1}+\frac{1}{4} \underline{y}_{1}+\frac{1}{4} \bar{y}_{1}$.

Taking into account that

$([0.8,1],[0,0]) \geq_{Q}([0,0.5],[0,0.5]) \geq_{Q}([0,0.3],[0.2,0.7])$ it holds

$$
\begin{array}{r}
I V A I O W A_{\left[w, \leq_{Q}\right]}(([0,0.3],[0.2,0.7]),([0.8,1],[0,0]), \\
([0,0.5],[0,0.5]))=([0.12,0.475],[0.1,0.525]) .
\end{array}
$$

Remark 1: It is important to mention:

1) Due to the characteristics of the orders above, in general, it is not true that

$$
\begin{aligned}
& \operatorname{IVAIOW} A_{[w, \leq]}\left(z_{1}, \ldots, z_{n}\right) \\
& =\left(I V O W A_{\left[w, \leq_{\left.B_{1,2}\right]}\right]}\left(\mathbf{x}_{\mathbf{1}}, \ldots, \mathbf{x}_{\mathbf{n}}\right),\right. \\
& \left.I V O W A_{\left[w, \leq_{\left.B_{1,2}\right]}\right]}\left(\mathbf{y}_{\mathbf{1}}, \ldots, \mathbf{y}_{\mathbf{n}}\right)\right) .
\end{aligned}
$$

In fact, this is not necessarily true even if the order is $\leq_{B}$, with $B=\left\langle B_{1}, B_{2}, B_{1}, B_{2}\right\rangle$. For example, consider the weight vector $w=(0.2,0.5,0.3)$ and the $\leq_{\Pi_{1}}$ order on $\mathcal{L}_{I V}([0,1])$. Taking into account that $([0.2,0.8],[0,0]) \geq_{\Pi_{1}}([0.1,0.2],[0.4,0.8]) \geq_{\Pi_{1}}$ $([0,0.3],[0.5,0.7])$ (see item 3 of Example 3.3) it holds

$$
\begin{aligned}
& \operatorname{IVAIOWA_{[w,\mathrm {\Pi }_{1}]}}(([0.2,0.8],[0,0]) \\
& \begin{aligned}
([0,0.3],[0.5,0.7]) & ([0.1,0.2],[0.4,0.8])) \\
& =([0.09,0.35],[0.35,0.61])
\end{aligned}
\end{aligned}
$$

Similarly, since $[0.2,0.8] \geq_{\Pi_{1,2}}[0.1,0.2] \geq_{\Pi_{1,2}}[0,0.3]$ and $[0.5,0.7] \geq_{\Pi_{1,2}}[0.4,0.8] \geq_{\Pi_{1,2}}[0,0]$ with $\Pi_{1,2}=$ $\left\langle\Pi_{1}, \Pi_{2}\right\rangle$ then

$\operatorname{IVOW} A([0.2,0.8],[0,0.3],[0.1,0.2])=[0.09,0.35]$ and

$\operatorname{IVOWA}([0,0],[0.5,0.7],[0.4,0.8])=[0.3,0.54]$.

However, $\quad([0.09,0.35],[0.35,0.61])$ $([0.09,0.35],[0.3,0.54])$.

2) Due to the monotonicity of OWAs, if we apply fixed weights to $\underline{x}, \bar{x}, \underline{y}, \bar{y}$, we produce the corresponding intervals. For example, taking the weight vector $w=$ $(0.2,0.5,0.3)$, such that we have the following results:
- $O W A_{w}(0.2,0,0.1)=0.09$

- $O W A_{w}(0.8,0.3,0.2)=0.37$

- $O W A_{w}(0,0.5,0.4)=0.3$

- $O W A_{w}(0,0.7,0.8)=0.51$,

we observe that the IVAI-pair $([0.09,0.37],[0.3,0.51]) \neq([0.09,0.35],[0.4,0.6])$

In fact, we obtain the first one, by ordering the extreme values of the intervals and not the intervals (see Aumann [21]).

This, or some others approaches generalizing the theoretical results on Atanassov Intuitionistic Fuzzy Sets such as [22] would be another manners of generalizing OWA operators over IVAIFSs but in this work we focus on studying the first approach of OWA operator given in Def 4.1. However, this example lets some open questions: What are the differences of these definitions? Are there any relation between the orders? Are there any orders which generate the same OWA operator for different definitions?

Proposition 4.2: Let be the IVAIOWA operator associated with $\leq$ and $w$. Given an order $\leq_{B_{1,2}}$ on $L([0,1])$ there exist $w^{\prime}$ and $w^{\prime \prime}$ two permutations of the vector $w$ induced by $\leq_{B_{1,2}}$ such that

$$
\begin{aligned}
& I V A I O W A_{[w, \leq]}\left(z_{1}, \ldots, z_{n}\right) \\
& =\left(I V O W A_{\left[w^{\prime}, \leq_{\left.B_{1,2}\right]}\right.}\left(\mathbf{x}_{\mathbf{1}}, \ldots, \mathbf{x}_{\mathbf{n}}\right),\right. \\
& \left.\quad \operatorname{IVOW} A_{\left[w^{\prime \prime}, \leq_{\left.B_{1,2}\right]}\right]}\left(\mathbf{y}_{\mathbf{1}}, \ldots, \mathbf{y}_{\mathbf{n}}\right)\right) .
\end{aligned}
$$

Proof. Straight. Besides, if $\mathbf{x}_{(i)}$ is the $i$-th greatest element according to $\leq_{B_{1,2}}$ then $w_{i}^{\prime}=w_{j}$ where $j$ is the position of the IVAIF-pair whose first interval is $\mathbf{x}_{(i)}$ through the $\leq$ order of IVAIF-pairs, namely, $z_{(j)}=\left(\mathbf{x}_{(i)}, \mathbf{y}^{*}\right)$.

Analogously, if $\mathbf{y}_{(i)}$ is the $i$-th greatest element according to $\leq_{B_{1,2}}$ then $w_{i}^{\prime \prime}=w_{j}$ where $j$ is the position of the IVAIF-pair whose second interval is $\mathbf{y}_{(i)}$ through the $\leq$ order of IVAIFpairs, namely, $z_{(j)}=\left(\mathbf{x}^{*}, \mathbf{y}_{(i)}\right)$.

Corollary 4.3: Let be $\leq_{B}$ with $B=\left\langle B_{1}, B_{2}, B_{1}, B_{2}\right\rangle$ and $\leq_{B_{1,2}}$ the orders used in the Prop. 4.2. Then $w^{\prime}=w$ satisfies Eq. (4).

Proof. Remember that $\leq_{B}$ uses first $\leq_{B_{1,2}}$ on $L([0,1])$ to compare the membership interval so that the elements in the set $\left\{\mathbf{x}_{i} \mid i \in\{1, \ldots, n\}\right\}$ are ordered in a decreasing order.

OWA operators, with the standard order between real numbers, are a special class of aggregation functions. However, as it occurs with IVOWA operators ([9]), IVAIOWA operators do not necessarily satisfy monotonicity.

Example 4.4: Let $B=\left\langle B_{1}, B_{2}, B_{1}, B_{2}\right\rangle$ with $B_{1}\left(x_{1}, x_{2}\right)=$ $x_{1} x_{2}$ and $B_{2}\left(x_{1}, x_{2}\right)=\frac{x_{1}+x_{2}}{2}$.

Let $z_{1}=([0,1],[0,0]), z_{2}=([0.2,0.35],[0.4,0.6])$ and $w=(0.9,0.1)$.

Taking into account that $z_{2}>_{B} z_{1}$ it holds

$$
\operatorname{IVAIOW} A_{\left[w, \leq_{B}\right]}\left(z_{1}, z_{2}\right)=([0.18,0.415],[0.36,0.54]) .
$$

However, if we take $\tilde{z}_{1}$ given by $\tilde{z}_{1}=$ $([0.1,0.15],[0.7,0.8]) \quad>_{B} \quad z_{1}=([0,1],[0,0])$ it holds 
$z_{2} \geq_{B} \tilde{z}_{1}$ and consequently,

$$
I V A I O W A_{\left[w, \leq_{B}\right]}\left(\tilde{z}_{1}, z_{2}\right)=([0.19,0.33],[0.43,0.62]) .
$$

Since

$([0.19,0.33],[0.43,0.62])<_{B}([0.18,0.415],[0.36,0.54])$ the monotonicity of the operator does not hold.

For sake of simplicity, given $M$ a 4-aggregation function we write $M(z)$ where $z$ is the IVAIF-pair given by $z=$ $([\underline{x}, \bar{x}],[\underline{y}, \bar{y}])$ to denote the image of $M(\underline{x}, \bar{x}, 1-\underline{y}, 1-\bar{y})$.

Proposition 4.5: Let be $\leq_{Q}$ the admissible order generated as in the Example 3.3 then

$$
\begin{aligned}
Q_{i}\left(I V A I O W A_{\left[w, \leq_{Q}\right]}\left(z_{1}, \ldots, z_{n}\right)\right) \\
\quad=w_{1} Q_{i}\left(z_{(1)}\right)+\ldots+w_{n} Q_{i}\left(z_{(n)}\right),
\end{aligned}
$$

where $z_{(i)}$ denotes the $i$-th greatest IVAIF-pair of the inputs $\left(z_{1}, \ldots, z_{n}\right)$ through the order $\leq_{Q}$ on $\mathcal{L}_{I V}([0,1])$.

Proof. Straight.

Remark 2: In the Proposition 4.5, for $i=1$ we have

$$
\begin{aligned}
Q_{1}\left(I V A I O W A_{\left[w, \leq_{Q}\right]}\right. & \left.\left(z_{1}, \ldots, z_{n}\right)\right) \\
= & O W A_{w}\left(Q_{1}\left(z_{1}\right), \ldots, Q_{1}\left(z_{n}\right)\right) .
\end{aligned}
$$

However, this does not necessarily hold for any other index.

Proposition 4.6: Let $\leq_{Q}$ be the order generated as in the Example 3.3 and $w \in(0,1]^{n}$. An IVAIOWA operator on $\mathcal{L}_{I V}([0,1])$ associated with $\leq_{Q}$ and $w$ is an aggregation function.

Proof. In order to simplify notation, we assume that the IVAIFpairs $\left(z_{1}, \ldots, z_{n}\right)$ are ordered in a decreasing way with respect to the order $\leq_{Q}$, i.e., $z_{1} \geq_{Q} \quad z_{2} \geq_{Q} \quad \ldots \geq_{Q} \quad z_{n}$. Notice that since IVAIOWA operators are symmetric, we do not lose generality by this assumption.

The boundary conditions are straight, but the monotonicity of the function needs to be proven. Let us assume that the IVAIOWA is not monotone. Then, there exist $\hat{z}_{i}$ satisfying that $z_{i} \leq_{Q} \hat{z}_{i}$ and such that

$$
\begin{aligned}
I V A I O W A_{\left[w, \leq_{Q}\right]}\left(z_{1}, \ldots, z_{i}, \ldots, z_{n}\right) \\
\quad>_{Q} I V A I O W A_{\left[w, \leq_{Q}\right]}\left(z_{1}, \ldots, \hat{z}_{i}, \ldots, z_{n}\right) .
\end{aligned}
$$

If $z_{i}=\hat{z}_{i}$ then $I V A I O W A_{\left[w, \leq_{Q}\right]}\left(z_{1}, \ldots, z_{i}, \ldots, z_{n}\right)={ }_{Q}$ IV AIOW $A_{\left[w, \leq_{Q}\right]}\left(z_{1}, \ldots, \hat{z}_{i}, \ldots, z_{n}\right)$ so we require $z_{i}<_{Q}$ $\hat{z}_{i}$. There exist four different cases:

$$
\begin{aligned}
& \text { i) } Q_{1}\left(I V A I O W A_{\left[w, \leq_{Q}\right]}\left(z_{1}, \ldots, z_{i}, \ldots, z_{n}\right)\right) \\
& >Q_{1}\left(I V A I O W A_{\left[w, \leq_{Q}\right]}\left(z_{1}, \ldots, \hat{z}_{i}, \ldots, z_{n}\right)\right) \\
& \text { ii) } Q_{1}\left(I V A I O W A_{\left[w, \leq_{Q}\right]}\left(z_{1}, \ldots, z_{i}, \ldots, z_{n}\right)\right) \\
& =Q_{1}\left(I V A I O W A_{\left[w, \leq_{Q}\right]}\left(z_{1}, \ldots, \hat{z}_{i}, \ldots, z_{n}\right)\right) \text { and } \\
& Q_{2}\left(I V A I O W A_{\left[w, \leq_{Q}\right]}\left(z_{1}, \ldots, z_{i}, \ldots, z_{n}\right)\right) \\
& >Q_{2}\left(I V A I O W A_{\left[w, \leq_{Q}\right]}\left(z_{1}, \ldots, \hat{z}_{i}, \ldots, z_{n}\right)\right) . \\
& =Q_{1}\left(I V A I O W A_{\left[w, \leq_{Q}\right]}\left(z_{1}, \ldots, \hat{z}_{i}, \ldots, z_{n}\right)\right) \text { and } \\
& Q_{2}\left(I V A I O W A_{\left[w, \leq_{Q}\right]}\left(z_{1}, \ldots, z_{i}, \ldots, z_{n}\right)\right) \\
& =Q_{2}\left(I V A I O W A_{\left[w, \leq_{Q}\right]}\left(z_{1}, \ldots, \hat{z}_{i}, \ldots, z_{n}\right)\right) \text { and } \\
& Q_{3}\left(I V A I O W A_{\left[w, \leq_{Q}\right]}\left(z_{1}, \ldots, z_{i}, \ldots, z_{n}\right)\right) \\
& >Q_{3}\left(I V A I O W A_{\left[w, \leq_{Q}\right]}\left(z_{1}, \ldots, \hat{z}_{i}, \ldots, z_{n}\right)\right) .
\end{aligned}
$$

iv) $Q_{1}\left(I V A I O W A_{\left[w, \leq_{Q}\right]}\left(z_{1}, \ldots, z_{i}, \ldots, z_{n}\right)\right)$

$$
\begin{aligned}
& =Q_{1}\left(I V A I O W A_{\left[w, \leq_{Q}\right]}\left(z_{1}, \ldots, \hat{z}_{i}, \ldots, z_{n}\right)\right) \text { and } \\
& Q_{2}\left(I V A I O W A_{\left[w, \leq_{Q}\right]}\left(z_{1}, \ldots, z_{i}, \ldots, z_{n}\right)\right) \\
& =Q_{2}\left(I V A I O W A_{\left[w, \leq_{Q}\right]}\left(z_{1}, \ldots, \hat{z}_{i}, \ldots, z_{n}\right)\right) \text { and } \\
& Q_{3}\left(I V A I O W A_{\left[w, \leq_{Q}\right]}\left(z_{1}, \ldots, z_{i}, \ldots, z_{n}\right)\right) \\
& =Q_{3}\left(I V A I O W A_{\left[w, \leq_{Q}\right]}\left(z_{1}, \ldots, \hat{z}_{i}, \ldots, z_{n}\right)\right) \text { and } \\
& Q_{4}\left(I V A I O W A_{\left[w, \leq_{Q}\right]}\left(z_{1}, \ldots, z_{i}, \ldots, z_{n}\right)\right) \\
& >Q_{4}\left(I V A I O W A_{\left[w, \leq_{Q}\right]}\left(z_{1}, \ldots, \hat{z}_{i}, \ldots, z_{n}\right)\right) .
\end{aligned}
$$

We tackled them individually:

i) If $Q_{1}\left(I V A I O W A_{\left[w, \leq_{Q}\right]}\left(z_{1}, \ldots, z_{i}, \ldots, z_{n}\right)\right)>$ $Q_{1}\left(I V A I O W A_{\left[w, \leq_{Q}\right]}\left(z_{1}, \ldots, \hat{z}_{i}, \ldots, z_{n}\right)\right)$, then by the Remark 2,

$$
\begin{gathered}
Q_{1}\left(I V A I O W A_{\left[w, \leq_{Q}\right]}\left(z_{1}, \ldots, z_{i}, \ldots, z_{n}\right)\right)= \\
O W A\left(Q_{1}\left(z_{1}\right), \ldots, Q_{1}\left(z_{i}\right), \ldots, Q_{1}\left(z_{n}\right)\right)> \\
O W A\left(Q_{1}\left(z_{1}\right), \ldots, Q_{1}\left(\hat{z}_{i}\right), \ldots, Q_{1}\left(z_{n}\right)\right) \\
\quad=Q_{1}\left(I V A I O W A_{\left[w, \leq_{Q}\right]}\left(z_{1}, \ldots, \hat{z}_{i}, \ldots, z_{n}\right)\right) .
\end{gathered}
$$

By the increasing monotonicity of OWA operators this implies $Q_{1}\left(z_{i}\right)>Q_{1}\left(\hat{z}_{i}\right)$ which contradicts $z_{i} \leq_{Q} \hat{z}_{i}$.

ii) If $Q_{1}\left(I V A I O W A_{\left[w, \leq_{Q}\right]}\left(z_{1}, \ldots, z_{i}, \ldots, z_{n}\right)\right)=$ $Q_{1}\left(I V A I O W A_{\left[w,<_{Q}\right]}\left(z_{1}, \ldots, \hat{z}_{i}, \ldots, z_{n}\right)\right)$, then by Eq. (5), $Q_{1}\left(z_{i}\right)=Q_{1}\left(\hat{z}_{i}\right)$. Two cases can be further discriminated:

- If the order of the IVAIF-pairs has not changed, by the Proposition 4.6, then

$$
\begin{gathered}
Q_{2}\left(I V A I O W A_{\left[w, \leq_{Q}\right]}\left(z_{1}, \ldots, z_{i}, \ldots, z_{n}\right)\right)= \\
w_{1} Q_{2}\left(z_{1}\right)+\ldots+w_{i} Q_{2}\left(z_{i}\right)+\ldots w_{n} Q_{2}\left(z_{n}\right)> \\
w_{1} Q_{2}\left(z_{1}\right)+\ldots+w_{i} Q_{2}\left(\hat{z}_{i}\right)+\ldots w_{n} Q_{2}\left(z_{n}\right)= \\
Q_{2}\left(I V A I O W A_{\left[w, \leq_{Q}\right]}\left(z_{1}, \ldots, \hat{z}_{i}, \ldots, z_{n}\right)\right),
\end{gathered}
$$

which implies $Q_{2}\left(z_{i}\right)>Q_{2}\left(\hat{z}_{i}\right)$, in contradiction with $z_{i} \leq_{Q} \hat{z}_{i}$.

- If the order of the IVAIF-pairs has changed $r$ positions, then it holds true

$$
Q_{1}\left(\hat{z}_{i}\right)=Q_{1}\left(z_{i-r}\right)=\ldots=Q_{1}\left(z_{i-1}\right)=Q_{1}\left(z_{i}\right)
$$

and

$$
Q_{2}\left(\hat{z}_{i}\right) \geq Q_{2}\left(z_{i-r}\right) \geq \ldots \geq Q_{2}\left(z_{i-1}\right) \geq Q_{2}\left(z_{i}\right) .
$$

However,

$$
\begin{aligned}
& Q_{2}\left(I V A I O W A_{\left[w, \leq_{Q}\right]}\left(z_{1}, \ldots, z_{i}, \ldots, z_{n}\right)\right) \\
= & w_{1} Q_{2}\left(z_{1}\right)+\ldots+w_{i} Q_{2}\left(z_{i}\right)+\ldots+w_{n} Q_{2}\left(z_{n}\right) \\
> & w_{1} Q_{2}\left(z_{1}\right)+\ldots+w_{i-r} Q_{2}\left(\hat{z}_{i}\right)+w_{i-(r-1)} Q_{2}\left(z_{i-r}\right) \\
+ & \ldots+w_{i} Q_{2}\left(z_{i-1}\right)+w_{i+1} Q_{2}\left(z_{i+1}\right)+\ldots+w_{n} Q_{2}\left(z_{n}\right) \\
= & Q_{2}\left(I V A I O W A_{\left[w, \leq_{Q}\right]}\left(z_{1}, \ldots, \hat{z}_{i}, \ldots, z_{n}\right)\right) .
\end{aligned}
$$

This implies that

$$
\begin{gathered}
w_{i-r} Q_{2}\left(z_{i-r}\right)+w_{i-(r-1)} Q_{2}\left(z_{i-(r-1)}\right)+\ldots+w_{i} Q_{2}\left(z_{i}\right) \\
>w_{i-r} Q_{2}\left(\hat{z}_{i}\right)+w_{i-(r-1)} Q_{2}\left(z_{i-r}\right)+\ldots+w_{i} Q_{2}\left(z_{i-1}\right),
\end{gathered}
$$

which is in contradiction with (6).

Items iii) and iv) are similar to item ii). 


\section{UNBALANCED INTERVAL-VALUED INTUITIONISTIC OWA OPERATORS}

In Def. 4.1 IVAIOWA operators used a fixed weight vector for both membership and nonmembership degrees. This is certainly practical, but at some situations it might be desirable to treat them differently. In this section we study the extension of IVAIOWA operators to cope with different weight vectors for the membership and nonmembership degrees.

Definition 5.1: Let $\leq$ be an IVAIF-admissible order on $\mathcal{L}_{I V}([0,1])$ and let $w, v \in[0,1]^{n}$ with $w_{1}+\ldots+w_{n}=1$ and $v_{1}+\ldots+v_{n}=1$. An Unbalanced IVAIOWA operator associated with $w, v$ and $\leq$ is a mapping $\left(\mathcal{L}_{I V}([0,1])\right)^{n} \longrightarrow$ $(L([0,1]))^{2}$ given by

$$
\begin{aligned}
U I V A I O W & A_{[w, v, \leq]}\left(z_{1}, \ldots, z_{n}\right) \\
= & \left(\sum_{i=1}^{n} w_{i} \cdot\left[\underline{x}_{(i)}, \bar{x}_{(i)}\right], \sum_{i=1}^{n} v_{i} \cdot\left[\underline{y}_{(i)}, \bar{y}_{(i)}\right]\right),
\end{aligned}
$$

where $\left(\left[\underline{x}_{(i)}, \bar{x}_{(i)}\right],\left[\underline{y}_{(i)}, \bar{y}_{(i)}\right]\right)$ denotes the $i$-th greatest of the inputs $\left(z_{1}, \ldots, z_{n}\right)$ with respect to the order $\leq$ on $\mathcal{L}_{I V}([0,1])$ and the interval product and sum are the same as used in Definition 2.7.

Remark 3: IVAIOWA operators in Section 3 are particular instances of Unbalanced IVAIOWA operators with $w=v$.

Notice that if there exist an index $i$ such that $w_{i}<v_{i}$, then there is an index $j$ such that $w_{j}>v_{j}$.

Next, we study the conditions under which Unbalanced IVAIOWA operators are aggregation functions, that is:

- They satisfy the boundary conditions.

- They are monotonic.

- The co-domain is $\mathcal{L}_{I V}([0,1])$, i.e. , the image of $n$ IVAIFpairs is always an IVAIF-pair. This is satisfied if

$$
\sum_{i=1}^{n} w_{i} \bar{x}_{(i)}+\sum_{i=1}^{n} v_{i} \bar{y}_{(i)} \leq 1 .
$$

Theorem 5.1: Unbalanced IVAIOWA operators always satisfy the boundary conditions.

Proof. The boundary conditions imply that

$$
\begin{aligned}
\operatorname{UIVAIOW} A_{[w, v, \leq]} & (([1,1],[0,0]), \ldots,([1,1],[0,0])) \\
& =\left(\sum_{i=1}^{n} w_{i} \cdot[1,1], \sum_{i=1}^{n} v_{i} \cdot[0,0]\right) \\
& =([1,1],[0,0])
\end{aligned}
$$

which is satisfied due to $\sum_{i=1}^{n} w_{i}=1$, and

$$
\begin{aligned}
\operatorname{UIVAIOW} A_{[w, v, \leq]} & (([0,0],[1,1]), \ldots,([0,0],[1,1])) \\
& =\left(\sum_{i=1}^{n} w_{i} \cdot[0,0], \sum_{i=1}^{n} v_{i} \cdot[1,1]\right) \\
& =([0,0],[1,1]),
\end{aligned}
$$

which is satisfied due to $\sum_{i=1}^{n} v_{i}=1$.

By the Proposition 4.6, the IVAIOWA operators associated with orders $\leq_{Q}$ (as in the Example 3.3) and weight vectors $w=v$ satisfy monotonicity. This property is not guaranteed for weight vectors $w \neq v$.
Example 5.2: Let $w=(0.1,0.9)$, and $v=(0.9,0.1)$ and let the order $\leq_{Q}$ be given by the four aggregation functions that follow:

- $Q_{1}\left(\underline{x}_{1}, \bar{x}_{1}, \underline{y}_{1}, \bar{y}_{1}\right)=\frac{1}{4} \underline{x}_{1}+\frac{1}{4} \bar{x}_{1}+\frac{1}{4} \underline{y}_{1}+\frac{1}{4} \bar{y}_{1}$,

- $Q_{2}\left(\underline{x}_{1}, \bar{x}_{1}, \underline{y}_{1}, \bar{y}_{1}\right)=\bar{x}_{1}$,

- $Q_{3}\left(\underline{x}_{1}, \bar{x}_{1}, \underline{y}_{1}, \bar{y}_{1}\right)=\underline{y}_{1}$,

- $Q_{4}\left(\underline{x}_{1}, \bar{x}_{1}, \underline{y}_{1}, \bar{y}_{1}\right)=\overline{\bar{y}}_{1}$.

Then, although $([0,0],[0,0])<_{Q}([0.5,0.5],[0.5,0.5])$, it holds

$$
\begin{aligned}
& U I V A I O W A_{\left[w, v, \leq_{Q}\right]}(([0,0],[0,0]),([0,0],[1,1])) \\
& \quad=([0,0],[0.1,0.1])>([0.05,0.05],[0.55,0.55])
\end{aligned}
$$

$U I V A I O W A_{\left[w, v, \leq_{Q}\right]}(([0.5,0.5],[0.5,0.5]),([0,0],[1,1]))$.

Consequently, $U I V A I O W A_{\left[w, v, \leq_{Q}\right]}$ is not monotonic.

Proposition 5.3: Let be $\leq_{K}$ an order generated as in the Example 3.3. Then the following holds

$$
\begin{aligned}
& K_{i}\left(U I V A I O W A_{\left[w, v, \leq_{K}\right]}\left(z_{1}, \ldots, z_{n}\right)\right) \\
& \quad=w_{1} K_{i}\left(z_{(1)}\right)+\ldots+w_{n} K_{i}\left(z_{(n)}\right), \quad i \in\{1,2\} \text { and } \\
& \quad K_{j}\left(U I V A I O W A_{\left[w, v, \leq_{K}\right]}\left(z_{1}, \ldots, z_{n}\right)\right) \\
& \quad=w_{1} K_{j}\left(z_{(1)}\right)+\ldots+w_{n} K_{j}\left(z_{(n)}\right), \quad j \in\{3,4\} .
\end{aligned}
$$

Proof. Straight.

Proposition 5.4: Let be $\leq_{K}$ the order generated as in the Example 3.3, and let $w, v \in(0,1]^{n}$ with $w_{1}+\ldots+w_{n}=1$ and $v_{1}+\ldots+v_{n}=1$. Then the UIVAIOW $A_{\left[w, v, \leq_{K}\right]}$ operator satisfies monotonicity.

Proof. Considering the Proposition 5.3, the proof is almost analogous to that of the Proposition 4.6.

Next, we study when the image of Unbalanced IVAIOWA operators is guaranteed to be IVAIF-pairs, i.e., $\left(\sum_{i=1}^{n} w_{i} \bar{x}_{(i)}+\sum_{i=1}^{n} v_{i} \bar{y}_{(i)} \leq 1\right)$. Note that this is trivially satisfied with $w=v$, and in the remainder of this section we only consider $w \neq v$.

Besides, when $\bar{y}_{i}=1-\bar{x}_{i}$ the equation is reduced to

$$
\sum_{i=1}^{n} w_{i} \bar{x}_{(i)}+\sum_{i=1}^{n} v_{i}\left(1-\bar{x}_{(i)}\right) \leq 1
$$

which is equivalent to

$$
\left(\sum_{i=1}^{n} w_{i} \bar{x}_{(i)} \leq \sum_{i=1}^{n} v_{i} \bar{x}_{(i)}\right) .
$$

Proposition 5.5: Let be $\leq_{K}$ an order generated as in the Example 3.3 with $k_{1} \in(0,1)$, and let $w, v \in(0,1]^{n}$ with $w_{1}+\ldots+w_{n}=1$ and $v_{1}+\ldots+v_{n}=1$. Then, the operator $U I V A I O W A_{\left[w, v, \leq_{K}\right]}$ is not always an IVAIF-pair.

Proof. We define the set of indexes

$$
\begin{aligned}
I & =\left\{j \mid w_{j_{1}}=v_{j_{1}}\right\}, \\
J_{1} & =\left\{j \mid w_{j_{1}}<v_{j_{1}}\right\} \text { and } \\
J_{2} & =\left\{j \mid w_{j_{2}}>v_{j_{2}}\right\} .
\end{aligned}
$$

As $w \neq v$, we have $J_{1} \neq \emptyset$ and $J_{2} \neq \emptyset$. Let $j_{0}=\min J_{1} \cup J_{2}$. 
As $w, v$ sum 1 and $\left\{1, \ldots, j_{0}-1\right\} \subseteq I$, then

$$
\sum_{k=1}^{j_{0}-1} w_{k}=\sum_{k=1}^{j_{0}-1} v_{k} \quad \text { and } \quad \sum_{k=j_{0}}^{n} w_{k}=\sum_{k=j_{0}}^{n} v_{k} .
$$

Let us show that there are always $n$ IVAIF-pairs whose image does not satisfy Eq. (8). We separate the proof in two different cases.

- If $j_{0} \in J_{2}$, then $w_{j_{0}}>v_{j_{0}}$. We choose the IVAIF-pairs

$$
\begin{aligned}
& \text { i) } z_{i}=([1 ; 1],[0 ; 0]) \text { for } i \in\left\{1, \ldots, j_{0}\right\} \text {; } \\
& \text { ii) } z_{i}=([0 ; 0],[1 ; 1]) \text { for } i \in\left\{j_{0}+1, \ldots, n\right\} \text {. }
\end{aligned}
$$

These IVAIF-pairs are top and bottom in $\mathcal{L}_{I V}([0,1])$ and they are ordered in a decreasing order. Since $\bar{x}_{(i)}=0$ for $i \in\left\{j_{0}+1, \ldots, n\right\}$ Eq. (8) results it

$$
\sum_{i=1}^{j_{0}} w_{i} \leq \sum_{i=i}^{j_{0}} v_{i}
$$

However, this only holds if

$$
\sum_{i=1}^{j_{0}-1} w_{i}+w_{j_{0}} \leq \sum_{i=i}^{j_{0}-1} v_{i}+v_{j_{0}}
$$

By Eq. (9), this is equivalent to $w_{j_{0}} \leq v_{j_{0}}$, which is in contradiction with $j_{0} \in J_{2}$.

- If $j_{0} \in J_{1}$, since $w_{j_{0}}<v_{j_{0}}$ then

$$
\sum_{i=j_{0}+1}^{n} w_{k}>\sum_{i=j_{0}+1}^{n} v_{k}
$$

We choose the IVAIF-pairs

i) $z_{i}=([1,1],[0,0])$ for $i \in\left\{1, \ldots, j_{0}-1\right\}$;

ii) $z_{j_{0}}=([0.4,0.4],[0.6,0.6])$;

iii) $z_{i}=([0, a],[0,1-a])$ for $i \in\left\{j_{0}+1, \ldots, n\right\}$, with $a \in(0.4,1]$ satisfying $a<\frac{0.4}{k_{1}}$ (this can hold as $\left.k_{1} \neq 1\right)$.

Notice that the IVAIF-pairs are ordered in a decreasing order. From 1 to $j_{0}-1$ the IVAIF-pairs are the top. Besides $z_{j_{0}}>z_{j_{0}+1}$ since $K_{1}([0.4,0.4],[0.6,0.6])=$ $0.4+k_{1}(0.4-0.4)=0.4$ and $K_{1}([0, a],[0,1-a])=$ $0+k_{1}(a-0)=k_{1} a$ but $k_{1} a<k_{1} \frac{0.4}{k_{1}}=0.4$. As the last ones are equal they are also ordered.

These IVAIF-pairs do not satisfy Eq. (8).

$$
\begin{aligned}
\sum_{k=1}^{j_{0}-1} w_{k}+0.4 w_{j_{0}} & +\sum_{k=j_{0}+1}^{n} a w_{k} \\
& \leq \sum_{k=1}^{j_{0}-1} v_{k}+0.4 v_{j_{0}}+\sum_{k=j_{0}+1}^{n} a v_{k} .
\end{aligned}
$$

First, by Eq.(9) the expression is reduced to

$$
0.4 w_{j_{0}}+\sum_{k=j_{0}+1}^{n} a w_{k} \leq 0.4 v_{j_{0}}+\sum_{k=j_{0}+1}^{n} a v_{k} .
$$

The expression can be rewritten

$$
\begin{aligned}
0.4 \sum_{k=j_{0}}^{n} w_{k}+(a & -0.4) \sum_{k=j_{0}+1}^{n} w_{k} \\
& \leq 0.4 \sum_{k=j_{0}}^{n} v_{k}+(a-0.4) \sum_{k=j_{0}+1}^{n} v_{k}
\end{aligned}
$$

if and only if

$$
(a-0.4) \sum_{k=j_{0}+1}^{n} w_{k} \leq(a-0.4) \sum_{k=j_{0}+1}^{n} v_{k},
$$

where the equivalence is due to Eq. (9).

Since $(a-0.4)>0$, the expression is reduced to

$$
\sum_{k=j_{0}+1}^{n} w_{k} \leq \sum_{k=j_{0}+1}^{n} v_{k}
$$

which is in contradiction with Eq. (10).

Consequently, the image of the $n$ IVAIF-pairs is not an IVAIF-pair and Unbalanced IVAIOWA operators could not be an aggregation function (since the domain and codomain are different sets).

Remark 4: In [20], it was proven that given $k_{1} \in(0,1]$, all the admissible orders on $L([0,1])$ with $k_{2}<k_{1}$ are equivalent. In this case, for the particular case of Unbalanced IVAIOWA operators, only $k_{1}=1$ can lead to well-defined operators. As a consequence, all the possible admissible orders are given by $\left(\mathbf{x}_{1}, \mathbf{y}_{\mathbf{1}}\right) \leq_{B}\left(\mathbf{x}_{\mathbf{2}}, \mathbf{y}_{\mathbf{2}}\right)$ if and only if

- $\left(\bar{x}_{1}<\bar{x}_{2}\right)$, or

- $\left(\bar{x}_{1}=\bar{x}_{2}\right.$ and $\left.\underline{x}_{1}<\underline{x}_{2}\right)$, or

- $\left(\bar{x}_{1}=\bar{x}_{2}, \underline{x}_{1}=\underline{x}_{2}\right.$, and

$$
K_{3}\left(\underline{x}_{1}, \bar{x}_{1}, \underline{y}_{1}, \bar{y}_{1}\right)>K_{3}\left(\underline{x}_{2}, \bar{x}_{2}, \underline{y}_{2}, \bar{y}_{2}\right) \text {, or }
$$

- $\left(\bar{x}_{1}=\bar{x}_{2}, \underline{x}_{1}=\underline{x}_{2}\right.$,

$$
\begin{aligned}
& K_{3}\left(\underline{x}_{1}, \bar{x}_{1}, \underline{y}_{1}, \bar{y}_{1}\right)=K_{3}\left(\underline{x}_{2}, \bar{x}_{2}, \underline{y}_{2}, \bar{y}_{2}\right) \text { and } \\
& \left.K_{4}\left(\underline{x}_{1}, \bar{x}_{1}, \underline{y}_{1}, \bar{y}_{1}\right) \geq K_{4}\left(\underline{x}_{2}, \bar{x}_{2}, \underline{y}_{2}, \bar{y}_{2}\right)\right)
\end{aligned}
$$

for some $k_{3}, k_{4} \in[0,1]$ with $k_{3} \neq k_{4}$.

We refer to these orders as $\leq_{O}$.

Next, we study the conditions under which the orders $\leq_{O}$ define an aggregation function.

Lemma 5.6: Let be $w, v \in\left(\mathbb{R}^{+}\right)^{n}$. Then the following statements are equivalent.

i) $\sum_{j=1}^{i} w_{j} \leq \sum_{j=1}^{i} v_{j}$ for all $i \in\{1, \ldots, n\}$.

ii) $\sum_{j=1}^{n} w_{j} t_{j} \leq \sum_{j=1}^{n} v_{j} t_{j}$ for all $t_{j} \in[0,1]$ such that $t_{1} \geq$ $t_{2} \geq \ldots \geq t_{n} \geq 0$.

Proof. We first prove that i) implies ii). As

$$
w_{1} \leq v_{1} \text { then } a_{1} w_{1} \leq a_{1} v_{1} \text { for all } a_{1} \geq 0 \text {. }
$$

$w_{1}+w_{2} \leq v_{1}+v_{2}$ then

$$
a_{2}\left(w_{1}+w_{2}\right) \leq a_{2}\left(v_{1}+v_{2}\right) \text { for all } a_{2} \geq 0 \text {. }
$$


$w_{1}+\ldots+w_{n} \leq v_{1}+\ldots+v_{n}$ then

$a_{n}\left(w_{1}+\ldots+w_{n}\right) \leq a_{n}\left(v_{1}+\ldots+v_{n}\right)$ for all $a_{n} \geq 0$.

If we sum

$$
\begin{array}{r}
\left(a_{1}+\ldots+a_{n}\right) w_{1}+\left(a_{2}+\ldots+a_{n}\right) w_{2}+\ldots+a_{n} w_{n} \\
\leq\left(a_{1}+\ldots+a_{n}\right) v_{1}+\left(a_{2}+\ldots+a_{n}\right) v_{2}+\ldots+a_{n} v_{n} \\
\text { for all } a_{1}, \ldots, a_{n} \geq 0 .
\end{array}
$$

Taking $t_{1}=\left(a_{1}+\ldots+a_{n}\right), t_{2}=\left(a_{2}+\ldots+a_{n}\right), \ldots, t_{n}=$ $a_{n}$ it satisfies ii).

To prove that ii) implies i), given $i \in\{1, \ldots, n\}$ take $t_{1}=$ $t_{2}=\ldots=t_{i}=1$ and $t_{i+1}=t_{i+2}=\ldots=t_{n}=0$.

Finally, we have the following characterization of Unbalanced IVAIOWA operators.

Theorem 5.7: Let $w, v \in[0,1]^{n}$ with $w_{1}+\ldots+w_{n}=1$ and $v_{1}+\ldots+v_{n}=1$. Then the following statements are equivalent:

i) Unbalanced IVAIOWA operators associated with $w, v$ and the orders $\leq_{O}$ is an aggregation function;

ii) $\sum_{i=1}^{n} w_{i} t_{i} \leq \sum_{i=1}^{n} v_{i} t_{i}$ for all $i=1, \ldots, n$ for all $t_{i} \in[0,1]$ such that $t_{1} \geq t_{2} \geq \ldots \geq t_{n} \geq 0$.

Proof. Let us show that i) implies ii). Suppose Unbalanced IVAIOWA operator is well defined. Then it satisfies Eq.(8) for the right endpoints of intervals $\sum_{i=1}^{n} w_{i} \bar{x}_{(i)} \leq \sum_{i=1}^{n} v_{i} \bar{x}_{(i)}$. Taking $t_{i}=\bar{x}_{(i)}$, it satisfies ii) since $\bar{x}_{(i)} \geq \bar{x}_{(j)}$ (because the order used is $\leq_{O}$ ).

Finally let us show that ii) implies i). First of all ii) can be rewritten as

$$
\begin{aligned}
& \sum_{i=1}^{n}\left(w_{i}-v_{i}\right) t_{i} \leq 0, \text { for all } t_{i} \in[0,1] \\
& \quad \text { such that } t_{1} \geq t_{2} \geq \ldots \geq t_{n} \geq 0 .
\end{aligned}
$$

Let $z_{i}=\left(\mathbf{x}_{i}, \mathbf{y}_{i}\right), i=1, \ldots, n$, be $n$ IVAIF-pairs. The expression of Unbalanced IVAIOWA operator associated with $w, v$ and one order $\leq_{O}$ is an aggregation function.

$$
\begin{aligned}
& U I V \operatorname{AIOW} A_{\left[w, v, \leq_{o}\right]}\left(z_{1}, \ldots, z_{n}\right) \\
& \quad=\left(\sum_{i=1}^{n} w_{i} \cdot\left[\underline{x}_{(i)}, \bar{x}_{(i)}\right], \sum_{i=1}^{n} v_{i} \cdot\left[\underline{y}_{(i)}, \bar{y}_{(i)}\right]\right)=(\mathbf{x}, \mathbf{y})
\end{aligned}
$$

where $\bar{x}_{(1)} \geq \bar{x}_{(2)} \geq \ldots \geq \bar{x}_{(n)}$ due to the order $\leq_{O}$. Considering that $\bar{x}_{(i)}+\bar{y}_{(i)} \leq 1$ and $v_{1}+v_{2}+\ldots+v_{n}=1$ then

$$
\begin{aligned}
& \bar{x}+\bar{y}= \\
& w_{1} \bar{x}_{(1)}+w_{2} \bar{x}_{(2)}+\ldots+w_{n} \bar{x}_{(n)}+v_{1} \bar{y}_{(1)}+v_{2} \bar{y}_{(2)}+\ldots+v_{n} \bar{y}_{(n)} \\
& \leq w_{1} \bar{x}_{(1)}+w_{2} \bar{x}_{(2)}+\ldots+w_{n} \bar{x}_{(n)} \\
& +v_{1}\left(1-\bar{x}_{(1)}\right)+v_{2}\left(1-\bar{x}_{(2)}\right)+\ldots+v_{n}\left(1-\bar{y}_{(n)}\right) \\
& =1+\left(w_{1}-v_{1}\right) \bar{x}_{1}+\left(w_{2}-v_{2}\right) \bar{x}_{2}+\ldots+\left(w_{n}-v_{n}\right) \bar{x}_{n} \leq 1 \text {, }
\end{aligned}
$$

where the last inequality is due to Eq. 11).

Corollary 5.8: Let $w, v \in(0,1]^{n}$ with $w_{1}+\ldots+w_{n}=1$ and $v_{1}+\ldots+v_{n}=1$. Then the following statements are equivalent: i) Unbalanced IVAIOWA operators associated with $w, v$ and the orders $\leq_{O}$;

ii) $\sum_{j=1}^{i} w_{j} \leq \sum_{j=1}^{i} v_{j}$ for all $i \in\{1, \ldots, n\}$

Proof. Straight by the Lemma 5.6 and the Theorem 5.7.

Example 5.9: Take $w=(0.3,0.2,0.5), v=(0.5,0.1,0.4)$ and the composed lexicographic 2 order $\leq_{O}$ which corresponds to $k_{1}=k_{3}=1$ and $k_{2}=k_{4}=0$. Then for $z_{1}=$ $([0,0.3],[0.5,0.7]), \quad z_{2}=([0.2,0.8],[0,0.2])$ and $z_{3}=$ $([0.1,0.2],[0.75,0.8])$, since $z_{2} \geq_{O} z_{1} \geq_{O} z_{3}$, it holds

$\operatorname{UIVAIOW} A_{\left[w, v, \leq_{o}\right]}\left(z_{1}, z_{2}, z_{3}\right)=([0.11,0.4],[0.35,0.49])$, which is an IVAIF-pair.

\section{Discrete InTERVAL-VALUED ATANASSOV INTUITIONISTIC CHOQUET INTEGRAL}

In this section, using IVAIF-admissible orders we define discrete Choquet integrals for IVAIFS.

Definition 6.1: Let $m$ be a fuzzy measure of a non-empty finite universe $U=\left\{u_{1}, \ldots, u_{n}\right\} \neq \emptyset$. The discrete Choquet integral of $G$ (an IVAIFS), with respect to an admissible order $\leq$ on $\mathcal{L}_{I V}([0,1])$ is given by

$$
\begin{aligned}
C_{[m, \leq]}(G)=\sum_{i=1}^{n}( & G\left(u_{\sigma(i)}\right) m\left(\left\{u_{\sigma(i)}, \ldots, u_{\sigma(n)}\right\}\right) \\
& \left.-G\left(u_{\sigma(i)}\right) m\left(\left\{u_{\sigma(i+1)}, \ldots, u_{\sigma(n)}\right\}\right)\right)
\end{aligned}
$$

where $\sigma:\{1, \ldots, n\} \longrightarrow\{1, \ldots, n\}$ is a permutation such that $G\left(u_{\sigma(1)}\right) \leq G\left(u_{\sigma(2)}\right) \leq \ldots \leq G\left(u_{\sigma(n)}\right)$ and $m\left(\left\{u_{\sigma(n+1)}, u_{\sigma(n)}\right\}\right)=0$, by convention.

If $G\left(u_{i}\right)=\left(\mathbf{x}_{i}, \mathbf{y}_{i}\right)$ for all $i=1, \ldots, n$ and $m_{i}=$ $m\left(\left\{u_{\sigma(i)}, \ldots, u_{\sigma(n)}\right\}\right)-m\left(\left\{u_{\sigma(i+1)}, \ldots, u_{\sigma(n)}\right\}\right)$ for $i=$ $1, \ldots, n$, the expression can be rewritten as

$$
\begin{aligned}
& C_{[m, \leq]}(G)= \\
& \left(\left[\sum_{i=1}^{n} \underline{x}_{\sigma(i)} m_{i}, \sum_{i=1}^{n} \bar{x}_{\sigma(i)} m_{i}\right],\left[\sum_{i=1}^{n} \underline{y}_{\sigma(i)} m_{i}, \sum_{i=1}^{n} \bar{y}_{\sigma(i)} m_{i}\right]\right) .
\end{aligned}
$$

Remark 5: If we use a symmetric fuzzy measure [23] in the integral Choquet for IVAIFS, we also recover IVAIOWA operators defined in Section 3.

Remember that if the order used is $\leq_{Q}$ as in the Example 3.3 then IV AIOWA operators are monotonic. However, this is not true in Choquet Integrals as proves the next example.

Example 6.1: Let $U=\left\{u_{1}, u_{2}\right\}$ and $G$ be an IVAIFS such that $G\left(u_{1}\right)=([0,0.3],[0.1,0.2])$ and $G\left(u_{2}\right)=$ $([0.1,0.5],[0,0.4])$.

Let $m$ be the fuzzy measure given for each $V \subset U$ by

$$
m(V)=\left(\frac{\sum_{u_{i} \in V} \underline{x}_{i}+\bar{x}_{i}+\underline{y}_{i}+\bar{y}_{i}}{\sum_{u_{j} \in U} \underline{x}_{j}+\bar{x}_{j}+\underline{y}_{j}+\bar{y}_{j}}\right)^{2}
$$

where $G\left(u_{i}\right)=\left(\left[\underline{x}_{i}, \bar{x}_{i}\right],\left[\underline{y}_{i}, \bar{y}_{i}\right)\right]$.

Consider the order $\leq \Pi_{\Pi_{2}}$, namely, the composed lexicographic 2 order:

$\left(\mathbf{x}_{1}, \mathbf{y}_{1}\right) \leq_{\Pi_{2}}\left(\mathbf{x}_{2}, \mathbf{y}_{2}\right)$ if and only if

- $\left(\bar{x}_{1}<\bar{x}_{2}\right)$, or 
- $\left(\bar{x}_{1}=\bar{x}_{2}\right.$ and $\left.\underline{x}_{1}<\underline{x}_{2}\right)$, or

- $\left(\bar{x}_{1}=\bar{x}_{2}, \underline{x}_{1}=\underline{x}_{2}\right.$, and $\left.\bar{y}_{1}>\bar{y}_{1}\right)$, or

- $\left(\bar{x}_{1}=\bar{x}_{2}, \underline{x}_{1}=\underline{x}_{2}, \bar{y}_{1}=\bar{y}_{1}\right.$ and $\left.\bar{y}_{1} \geq \bar{y}_{1}\right)$

The Choquet integral of $G$ is

$$
\begin{gathered}
C_{\left[m, \leq_{\Pi_{2}}\right]}(G)=([0,0.3] \cdot(1-0.39)+[0.1,0.5] \cdot(0.39-0), \\
[0.1,0.2] \cdot(1-0.39)+[0,0.4] \cdot(0.39-0))= \\
([0.039,0.378],[0.061,0.278]) .
\end{gathered}
$$

If we take the IVAIF-pair $G^{\prime}\left(u_{2}\right)=([0,0.6],[0,0]) \geq \Pi_{2}$ $([0.1,0.5],[0,0.4])=G\left(u_{2}\right)$, then

$$
\begin{gathered}
C_{\left[m, \leq_{\Pi_{2}}\right]}\left(G^{\prime}\right)=([0,0.3] \cdot(1-0.25)+[0,0.6] \cdot(0.25-0), \\
[0.1,0.2] \cdot(1-0.25)+[0,0] \cdot(0.25-0))= \\
([0,0.375],[0.075,0.15]) .
\end{gathered}
$$

But

$([0,0.375],[0.075,0.15])<_{\Pi_{2}}([0.039,0.378],[0.061,0.278])$ and the Choquet integral is not monotonic.

Open Problem: When are discrete Choquet integrals monotone? Notice that they depend on the measure $m$, that can depend on the values of the inputs as in the Example 6.1.

\section{APPLICATION TO MULTI-EXPERT DECISION MAKING}

Multi-expert decision making consists of choosing an alternative out of a given set $U=\left\{u_{1}, \ldots, u_{p}\right\},(p \geq 2)$, according to the pairwise preferences given by some experts $E=$ $\left\{e_{1}, \ldots, e_{n}\right\},(n>2)$. The concordances and discordances of such preferences must be taken into account in the process of choosing the best-possible alternative. Frequently, experts have difficulties in defining and quantifying their preferences between pairs of alternatives. In order to solve these difficulties, decision-making algorithms allow increasingly elaborated expressions of preference [24], [25] . In this work we consider the case where the expression of the preference of the experts is given by IVAIF-pairs.

\section{A. Algorithms for interval-valued intuitionistic preference re- lations}

B. Algorithms for interval-valued intuitionistic preference relations

An interval-valued Atanassov intuitionistic fuzzy preference relation $R_{\mathrm{IVAIF}}$ on $U$ is a mapping $U \times U \longrightarrow \mathcal{L}_{I V}([0,1])$ such that $R_{\mathrm{IVAIF}}\left(u_{i}, u_{j}\right)$ represents the desirability of the alternative $u_{i}$ over alternative $u_{j}$. For each of such IVAIF-pairs the first interval denotes the degree of preference of $u_{i}$ over $u_{j}$, while the second one represents the non-preference of $u_{i}$ over $u_{j}$.

A multi-expert decision making algorithm takes as input the opinion of multiple experts. Each of such experts $e \in$ $E$ expresses his preferences as an interval-valued Atanassov intuitionistic fuzzy relation, which is denoted $R_{\mathrm{IVAIF}}^{e}$.

$$
R_{\mathrm{IVAIF}}^{e}=\left(\begin{array}{cccc}
- & z_{12} & \ldots & z_{1 p} \\
z_{21} & - & \ldots & z_{2 p} \\
\vdots & \ddots & \ddots & \vdots \\
z_{p 1} & \ldots & z_{p(p-1)} & -
\end{array}\right)
$$

Note that the elements in the main diagonal are unset, since they represent preference of each alternative over itself. For the sake of simplicity, we take $z_{i j}=\left(\mathbf{x}_{i j_{e}}, \mathbf{y}_{i j_{e}}\right)$.

The method for choosing one alternative $u^{\prime} \in U$ is depicted in Algorithm 1. The a priori information consists of the set of alternatives $U$, the preference relations generated by the experts and the weight vector and admissible orders used by the IVAIOWA operator and IVAIF Choquet integral, respectively. The result is expressed as the preferred alternative $u^{\prime}$. The algorithm has two main phases, namely information fusion and exploitation. In the information fusion phase, the so-called collective preference relation is created. This relation fusions the preferences of each of the experts for each pair of alternatives. In the exploitation phase the algorithm models the global desirability of each of the alternatives. In order to do so, it computes a fuzzy measure for each of the alternatives (i.e. for each row in the collective preference relation). This fuzzy measure is further used in an IVAIF Choquet integral to produce a global desirability value $z_{i}$, which recalls the preference of the alternative $u_{i}$ over all of the other alternatives. Finally, the alternative $u_{i}$ whose $z_{i}$ is maximum is taken as preferred alternative (if more than one alternative produces such maximum, any of them can be taken as preferred alternative).

Data: A set of alternatives $U$, a set of relations $R_{\mathrm{IVAIF}}^{e}$, a weight vector $w \in(0,1]^{n}$, an IVAIF-admissible order $\leq_{Q}$

Result: A preferred alternative $u^{\prime} \in U$

// 1- Information fusion: Creating a collective preference relation

for each position $(i, j) \in U \times U$ do

$R_{\mathrm{IVAIF}}^{c}(i, j) \leftarrow$ $\quad I V A I O W A_{\left[w, \leq_{Q}\right]}\left(R_{\mathrm{IVAIF}}^{e 1}(i, j), \ldots, R_{\mathrm{IVAIF}}^{e_{n}}(i, j)\right)$;

end

// 2- Exploitation

for each row $i$ of $R_{\text {IVAIF }}^{c}$ do

// 2.1- Build the fuzzy measures $m_{i}$ $A_{i}=\mathcal{P}(\{1, \ldots, p\} \backslash i)$;

for each $A^{\prime} \in A_{i}$ do $m_{i}\left(A^{\prime}\right) \leftarrow$ $\left(\frac{\sum_{j \in A^{\prime}} \underline{x}_{i j}+\bar{x}_{i j}+\left(1-\underline{y}_{i j}\right)+\left(1-\bar{y}_{i j}\right)}{\sum_{\substack{l=1 \\ l \neq i}}^{p} \underline{x}_{i l}+\bar{x}_{i l}+\left(1-\underline{y}_{i l}\right)+\left(1-\bar{y}_{i l}\right)}\right)^{2}$

end

// 2.2- Aggregate the matrix row-wise

for each row $i$ of $R_{I V A I F}^{c}$ do

$\mid z_{i} \leftarrow C_{\left[m_{i}, \leq_{Q}\right]}\left(R_{\mathrm{IVAIF}}^{c}(i, j)\right)$ with $j \in\{1, \ldots, n\} \backslash\{i\}$; end

// 2.3- Select the most preferred alternative

$u^{\prime} \leftarrow u_{k}$ such that $z_{k}$ is maximum;

Algorithm 1: First algorithm for multi-expert decision making using interval-valued Atanassov intuitionistic fuzzy preference relations.

The method in Algorithm 1 is fairly simple and powerful, but can also suffer from unexpected behaviours. This is due to the non-monotonicity of Choquet integrals through the IVAIFadmissible orders $\leq_{Q}$. Hence, an increase of the values in the $i$-th row of $R_{\mathrm{IVAIF}_{c}}$ might potentially lead to a reduction of the value $z_{i}$. Put to interpretable terms, this means that 
an increase of the preferences of a given alternative over the others can lead to a reduction of its global desirability. Although in some situations this fact might have no impact on the final choice, it is certainly undesirable. This issue is solved by the Algorithm 2. It modifies the exploitation phase of Algorithm 1 by aggregating the fuzzy $R_{\mathrm{IVAIF}}^{c}$ through an Unbalanced IVAIOWA operator. However, the orders must be restricted to $\leq_{O}$.

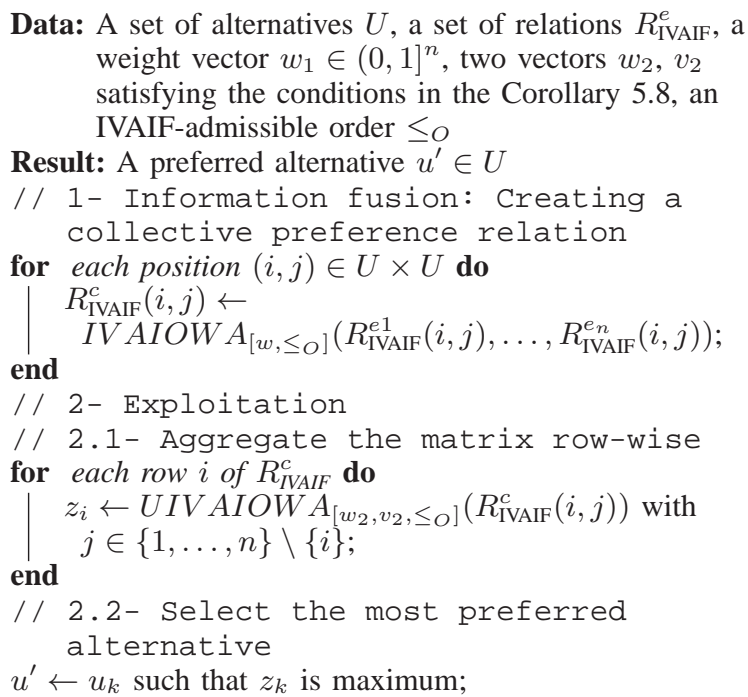

Algorithm 2: Second algorithm for multi-expert decision making using interval-valued Atanassov intuitionistic fuzzy preference relations.

Note that although in Algorithm 2 the non-monotonicity of Choquet integral is solved, it impose the order to be in the class of $\leq_{O}$ which is more restrictive than $\leq_{Q}$ class.

\section{Example of multi-expert decision making}

Let $\left\{z_{1}, \ldots, z_{4}\right\}$ represent four alternatives on which three experts provide their personal preferences. The preference relations obtained for each of the experts are depicted in Table I.

We intend to take a decision on the best possible option using the weight vector $w=(0.3,0.4,0.3)$, which gives more importance to the intermediate IVAIF-pair, i.e., we give more importance to the neutral expert (neither the optimistic, nor the pessimistic).

The collective matrix if the Lexicographic- 1 order $\left(\leq_{\Pi_{1}}\right)$ is chosen in Algorithm 1, is given in Table II.

After exploitation phase, the global desirability values are

$$
\begin{aligned}
& z_{1}=([0.4299,0.5592],[0.231,0.355]), \\
& z_{2}=([0.3826,0.5162],[0.257,0.3448]), \\
& z_{3}=([0.3024,0.4201],[0.3272,0.4227]), \text { and } \\
& z_{4}=([0.3551,0.6636],[0.181,0.2692]) .
\end{aligned}
$$

In this way, through the Lexicographic-1 order, the preferred option is $u_{1}$, followed by $u_{2}, u_{4}$ and $u_{3}$. The question remains open on whether other orders would yield the same decision on the existing preferences. The algorithm has been repeated using
- $\leq_{\Pi_{2}}$ as in the Example 3.3.

- $\leq_{K}$ generated by $K_{1}, K_{2}, K_{3}, K_{4}$ with $k_{1}=k_{3}=\frac{3}{4}$ and $k_{2}=k_{4}=\frac{1}{4}$.

- $\leq_{Q}$ with $Q_{1}, Q_{2}, Q_{3}, Q_{4}$ given by

$$
\begin{aligned}
& Q_{1}\left(\underline{x}_{1}, \bar{x}_{1}, \underline{y}_{1}, \bar{y}_{1}\right)=\frac{1}{4} \underline{x}_{1}+\frac{1}{4} \bar{x}_{1}+\frac{1}{4} \underline{y}_{1}+\frac{1}{4} \bar{y}_{1}, \\
& Q_{2}\left(\underline{x}_{1}, \bar{x}_{1}, \underline{y}_{1}, \bar{y}_{1}\right)=\frac{10}{20} \underline{x}_{1}+\frac{3}{20} \bar{x}_{1}+\frac{3}{20} \underline{y}_{1}+\frac{4}{20} \bar{y}_{1}, \\
& Q_{3}\left(\underline{x}_{1}, \bar{x}_{1}, \underline{y}_{1}, \bar{y}_{1}\right)=\frac{2}{10} \underline{x}_{1}+\frac{2}{10} \bar{x}_{1}+\frac{3}{10} \underline{y}_{1}+\frac{3}{10} \bar{y}_{1} \text { and } \\
& Q_{4}\left(\underline{x}_{1}, \bar{x}_{1}, \underline{y}_{1}, \bar{y}_{1}\right)=\frac{1}{10} \underline{x}_{1}+\frac{4}{10} \bar{x}_{1}+\frac{1}{10} \underline{y}_{1}+\frac{4}{10} \bar{y}_{1} .
\end{aligned}
$$

The global desirability of the alternatives with each of the orders is as follows:

- Order $\leq_{\Pi_{1}}: z_{1} \geq z_{2} \geq z_{4} \geq z_{3}$;

- Order $\leq_{\Pi_{2}}$ : $z_{4} \geq z_{1} \geq z_{2} \geq z_{3}$;

- Order $\leq_{K}: z_{1} \geq z_{4} \geq z_{3} \geq z_{2}$;

- Order $\leq_{Q}: z_{4} \geq z_{1} \geq z_{2} \geq z_{3}$.

So, depending on the chosen IVAIF-admissible order Alternatives 1 and 4 can be depicted. If non-monotonicity of IVAIF Choquet is affecting the result, we decide to run Algorithm 2. Taking the order $\leq_{\Pi_{2}}$ and the weight vectors $w_{2}=(0.3,0.2,0.5)$ and $v_{2}=(0.5,0.1,0.4)$ (which satisfy the conditions of Corollary 5.8). The final values of Unbalanced IVAIOWA operator are

$$
\begin{aligned}
& z_{1}=([0.4265,0.5640],[0.1930,0.3280]), \\
& z_{2}=([0.3460,0.4917],[0.2334,0.3252]), \\
& z_{3}=([0.3470,0.4397],[0.2445,0.3115]), \\
& z_{4}=([0.4455,0.6395],[0.1820,0.2868]) .
\end{aligned}
$$

Consequently, $u_{4}$ is preferred over all the other alternatives by Algorithm 2.

\section{CONCLUSIONS}

In this work we have analyzed the extension of OWA operators and discrete Choquet integral to cope with IVAIFSs. This has led to the proposal of novel definitions of IVAIOWA operators, Unbalanced IVAIOWA operators and IVAIF Choquet integrals. In the definition of these operators we have considered the possibility of choosing different weight vectors for the membership and non-membership. We have also studied the role of the IVAIF-admissible orders of IVAIF-pairs, more specifically the impact of such orders in the monotonicity of the IVAIOWA operators. For illustrative purposes, we have presented examples of application in the context of multiexpert decision making, considering two different algorithms in which the novel operators can take a relevant role.

\section{ACKNOWLEDGMENT}

This work has been partially supported by the project TIN2013-40765-P(Spain); the Research Services of the Universidad Publica de Navarra; the project APVV-14-0013; and the Brazilian funding agency $\mathrm{CNPq}$ under the Processes 406503/2013-3 and 307681/2012-2. 


$$
\begin{aligned}
& R_{I V A I F}^{e_{1}}=\left(\begin{array}{cccc}
- & {[0.38,0.38],[0.57,0.62]} & {[0.64,0.68],[0.24,0.3]} & {[0.49,0.71],[0.14,0.22]} \\
{[0.3,1],[0,0]} & - & {[0.11,0.23],[0.64,0.68]} & {[0.2,0.3],[0,0.05]} \\
{[0,0.1],[0.6,0.68]} & {[0.16,0.27],[0.3,0.55]} & - & {[0.6,0.62],[0,0.1]} \\
{[0.28,0.76],[0.22,0.22]} & {[1,1],[0,0]} & {[0.07,0.29],[0.6,0.71]} & -
\end{array}\right) \\
& R_{I V A I F}^{e_{2}}=\left(\begin{array}{cccc}
- & {[0.3,0.46],[0,0.5]} & {[0.1,0.43],[0.5,0.57]} & {[0.86,0.92],[0,0.05]} \\
{[0.9,0.9],[0,0.1]} & - & {[0.14,0.36],[0.2,0.6]} & {[0.6,0.67],[0.1,0.21]} \\
{[0,0.1],[0.3,0.4]} & {[0.16,0.27],[0.7,0.73]} & - & {[0.4,0.5],[0.5,0.5]} \\
{[0.61,0.76],[0,0.11]} & {[0.5,0.6],[0.2,0.4]} & {[0.22,0.76],[0.1,0.18]} & -
\end{array}\right) \\
& R_{I V A I F}^{e_{3}}=\left(\begin{array}{cccc}
- & {[0.4,0.6],[0,0]} & {[0.71,0.83],[0,0.1]} & {[0.15,0.3],[0.48,0.6]} \\
{[0.9,0.9],[0,0]} & - & {[0.1,0.15],[0.7,0.84]} & {[0.3,0.4],[0.5,0.52]} \\
{[0.8,0.84],[0.06,0.1]} & {[0.26,0.6],[0.31,0.4]} & - & {[0.9,0.95],[0,0.02]} \\
{[0.12,0.46],[0,0.3]} & {[0.2,0.23],[0.7,0.74]} & {[0.74,0.93],[0,0]} & -
\end{array}\right)
\end{aligned}
$$

TABLE I

PREFERENCES RELATIONS GIVEN BY THE THREE EXPERTS

$$
R_{I V A I F}^{c}=\left(\begin{array}{cccc}
{[0.72,0.93],[0,0.04]} & {[0.362,0.47],[0.228,0.398]} & {[0.499,0.65],[0.246,0.321]} & {[0.499,0.65],[0.2,0.283]} \\
{[0.24,0.322],[0.318,0.394]} & {[0.19,0.369],[0.423,0.559]} & {[0.116,0.245],[0.526,0.704]} & {[0.36,0.451],[0.23,0.286]} \\
{[0.331,0.67],[0.088,0.211]} & {[0.56,0.609],[0.29,0.382]} & {[0.331,0.67],[0.22,0.285]} & {[0.63,0.683],[0.15,0.196]}
\end{array}\right)
$$

TABLE II

COLLECTIVE PREFERENCE RELATION USING LEXICOGRAPHIC-1 ORDER FOR FUSING THE INFORMATION.

\section{REFERENCES}

[1] R. Yager, "On ordered weighted averaging aggregation operators in multicriteria decisionmaking," Systems, Man and Cybernetics, IEEE Transactions on, vol. 18, no. 1, pp. 183-190, 1988.

[2] G. Choquet, "Theory of capacities," Annales de l Institut Fourier, 1953.

[3] K. Atanassov and G. Gargov, "Interval-valued intuitionistic fuzzy sets," Fuzzy Sets and Systems, vol. 31, no. 3, pp. 343-349, 1989.

[4] L. D. Miguel, H. Bustince, J. Fernandez, E. Induráin, A. Kolesárová, and R. Mesiar, "Construction of admissible linear orders for intervalvalued Atanassov intuitionistic fuzzy sets with an application to decision making," Information Fusion, vol. 27, pp. 189 - 197, 2016.

[5] E. Barrenechea, J. Fernandez, M. Pagola, F. Chiclana, and H. Bustince, "Construction of interval-valued fuzzy preference relations from ignorance functions and fuzzy preference relations. application to decision making," Knowledge-Based Systems, vol. 58, pp. 33 - 44, 2014

[6] Z. Xu, "A method based on distance measure for interval-valued intuitionistic fuzzy group decision making," Information Sciences, vol. 180, no. 1, pp. 181 - 190, 2010.

[7] G. Deschrijver, "Additive and multiplicative generators in interval-valued fuzzy set theory," Fuzzy Systems, IEEE Transactions on, vol. 15, no. 2, pp. 222-237, April 2007.

[8] J. Sanz, A. Fernandez, H. Bustince, and F. Herrera, "IVTURS: A linguistic fuzzy rule-based classification system based on a new intervalvalued fuzzy reasoning method with tuning and rule selection," Fuzzy Systems, IEEE Transactions on, vol. 21, no. 3, pp. 399-411, 2013.

[9] H. Bustince, M. Galar, B. Bedregal, A. Kolesárová, and R. Mesiar, "A new approach to interval-valued Choquet integrals and the problem of ordering in interval-valued fuzzy set applications," Fuzzy Systems, IEEE Transactions on, vol. 21, no. 6, pp. 1150-1162, 2013.

[10] L. A. Zadeh, "Fuzzy sets," Information and control, vol. 8, no. 3, pp. 338-353, 1965.

[11] H. Bustince, E. Barrenechea, M. Pagola, J. Fernandez, Z. Xu, B. Bedregal, J. Montero, H. Hagras, F. Herrera, and B. De Baets, "A historical account of types of fuzzy sets and their relationships," Fuzzy Systems, IEEE Transactions on, vol. 24, no. 1, pp. 179-194, 2016.

[12] K. T. Atanassov, "Intuitionistic fuzzy sets," Fuzzy Sets and Systems, vol. 20, no. 1, pp. 87-96, 1986.

[13] H. Bustince and P. Burillo, "Correlation of interval-valued intuitionistic fuzzy sets," Fuzzy Sets and Systems, vol. 74, no. 2, pp. 237 - 244, 1995.
[14] J.-Q. Wang, P. Wang, J. Wang, H.-Y. Zhang, and X.-H. Chen, "Atanassov's interval-valued intuitionistic linguistic multicriteria group decision-making method based on the trapezium cloud model," Fuzzy Systems, IEEE Transactions on, vol. 23, no. 3, pp. 542-554, 2015.

[15] P. Liu, "Some Hamacher aggregation operators based on the intervalvalued intuitionistic fuzzy numbers and their application to group decision making," Fuzzy Systems, IEEE Transactions on, vol. 22, no. 1, pp. 83-97, 2014.

[16] G. Beliakov, H. Bustince, and T. Calvo, A Practical Guide to Averaging Functions. Studies In Fuzziness and Soft Computing, Springer, 2016.

[17] T. Calvo, A. Kolesárová, M. Komorníková, and R. Mesiar, "Aggregation operators: properties, classes and construction methods," in Aggregation Operators, ser. Studies in Fuzziness and Soft Computing, T. Calvo, G. Mayor, and R. Mesiar, Eds. Physica-Verlag HD, 2002, vol. 97, pp. 3-104.

[18] M. Komorníková and R. Mesiar, "Aggregation functions on bounded partially ordered sets and their classification," Fuzzy Sets and Systems, vol. 175, no. 1, pp. 48-56, 2011.

[19] G. Beliakov, A. Pradera, and T. Calvo, Aggregation Functions: A Guide for Practitioners. Studies In Fuzziness and Soft Computing, Springer, 2007.

[20] H. Bustince, J. Fernandez, A. Kolesárová, and R. Mesiar, "Generation of linear orders for intervals by means of aggregation functions," Fuzzy Sets and Systems, vol. 220, pp. 69-77, 2013.

[21] R. J. Aumann, "Integrals of set-valued functions," Journal of Mathematical Analysis and Applications, vol. 12, no. 1 , pp. 1 - 12, 1965. [Online]. Available: http://www.sciencedirect.com/science/article/pii/0022247X65900491

[22] K. Atanassov, P. Vassilev, and R. Tsvetkov, Intuitionistic Fuzzy Sets, measures and integrals. Professor Marin Drinov Academic Publishing House, 2013.

[23] Z. Wang and G. Klir, Fuzzy Measure Theory. Plenum, New York, 1992.

[24] Z. Xu, "A C-OWA operator-based approach to decision making with interval fuzzy preference relation," International Journal of Intelligent Systems, vol. 21, no. 12, pp. 1289-1298, 2006.

[25] Z. Xu and X. Cai, "Incomplete interval-valued intuitionistic fuzzy preference relations," International Journal of General Systems, vol. 38, no. 8, pp. 871-886, 2009. 


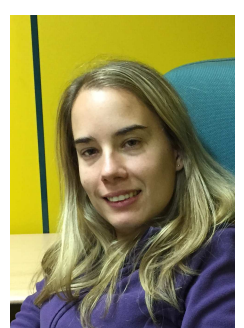

Laura De Miguel received the degree in mathematics from the University of Zaragoza, Spain, in 2012. She is currently a Phd student in the Department of Automatics and Computation, Public University of Navarra. Her areas of interest are fuzzy logics and their extensions related with concepts such as aggregation functions.

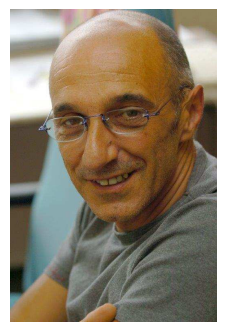

Humberto Bustince received the Ph.D. degree in mathematics from the Public University of Navarra, Pamplona, Spain, in 1994. He is currently a Full Professor with the Department of Automatics and Computation, Public University of Navarra. He is the author of more than 190 published original articles and is involved in teaching artificial intelligence for students of computer sciences. His research interests include fuzzy logic theory, extensions of fuzzy sets (type-2 fuzzy sets, interval-valued fuzzy sets, Atanassovs intuitionistic fuzzy sets), fuzzy measures, aggregation functions, and fuzzy techniques for image processing. Dr. Bustince is a board member of the European Society for Fuzzy Logic and Technology. He is the Editor-in-Chief of the Mathware and Soft Computing Magazine.

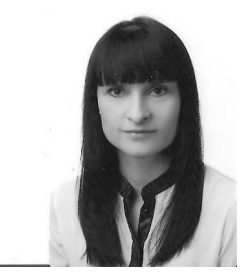

Barbara Pekala received the M.Sc. degree in mathematics in 1999 from University of Rzeszów (UR), Poland and the Ph.D. degree in mathematics in 2008 from AGH University of Science and Technology (Cracow, Poland). She is currently an Assistant Professor with the Faculty of Mathematics and Natural Sciences (UR). She is the author of more than 30 research papers. Her areas of interest are fuzzy relations, aggregation functions, preference structures and extensions of fuzzy sets. She is a member of Polish Mathematical Society.

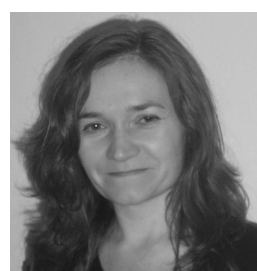

Urszula Bentkowska received the M.Sc. degree in mathematics in 1999 from University of Rzeszów (UR), Poland and the Ph.D. degree in mathematics in 2006 from AGH University of Science and Technology (Cracow, Poland). She is currently an Assistant Professor with the Faculty of Mathematics and Natural Sciences (UR). She is the author of more than 35 research papers. Her areas of interest are fuzzy relations and related concepts like aggregation functions, fuzzy connectives and moreover extensions of fuzzy sets. She is a member of Polish

Mathematical Society.

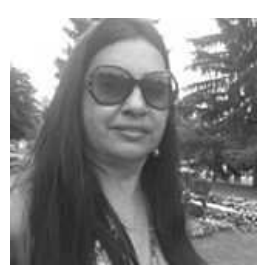

Ivanoska Da Silva is a Ph.D. student in Electrical Engineering and computer sciences of the Federal University of Rio Grande do Norte (UFRN), Natal, Brazil, since 2012. She is an administrator at the UFRN since 2004. Her research interests include: non-standard fuzzy sets theory, aggregation functions, and decision making.

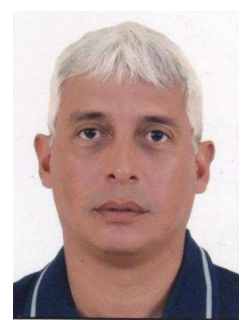

Benjamin Bedregal received the Ph.D. degree in computer sciences from the Federal University of Pernambuco (UFPE), Recife, Brazil, in 1996. In 1996, he became Assistant Professor at the Department of Informatics and Applied Mathematics, Federal University of Rio Grande do Norte (UFRN), Natal, Brazil, where he is currently a Full Professor. His research interests include: non-standard fuzzy sets theory, aggregation functions, clustering, decision making, fuzzy mathematics, and fuzzy automata.

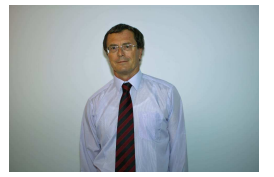

Radko Mesiar is head of the Department of Mathematics at Faculty of Civil Engineering, STU Bratislava. Graduated at Comenius University, Faculty of Mathematics and Physics, in 1974, PhD at the same faculty obtained in 1979 with $\mathrm{PhD}$ thesis 'Subadditive martingale processes'. Since 1978 member of the Department of Mathematics at Faculty of Civil Engineering, STU Bratislava. DSc since 1996 (in Czech Republic, Academy of Sciences). Associate professor since 1983, full professor since 1998. Fellow member of Czech Academy of Sciences, Institute of Information and Automation, Praha (Czech Republic, since 1995) and of IRAFM, University of Ostrava (Czech Republic, since 2006). Co-author of two scientific monographs (Triangular Norms, Kluwer, 2000; Aggregation Functions, Cambridge University Press, 2009), 5 edited volumes and author of more than 200 papers in WoS journals. Founder and organizer of conferences FSTA and AGOP.

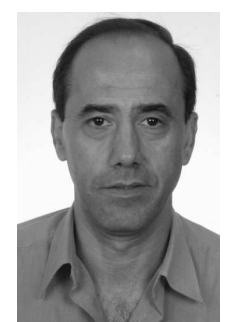

Gustavo Ochoa received the M.Sc. and Ph.D. degrees in mathematics from the University of Zaragoza, Spain, respectively in 1981 and 1984 . $\mathrm{He}$ is currently an Assistance Lecturer with the Department of Mathematics, Public University of Navarra, Pamplona, Spain. His research interests include group theory, representation theory and more recently fuzzy sets and aggregation functions applied to decision making and image processing. 